COMMUNICATIONS IN

ANALYSIS AND GEOMETRY

Volume 3, Number 3, 371-419, 1995

\title{
Uniton bundles
}

\section{Christopher Kumar ANAND ${ }^{1}$}

We show that a twistor construction of Hitchin and Ward can be adapted to study unitons (harmonic spheres in a unitary group). Specifically, we show that unitons are equivalent to holomorphic bundles with extra structure over a rational ruled surface with energy given by Chern class. This equivalence allows us to confirm the conjecture of Wood that unitons are rational. We also construct an example of a two uniton in $U(3)$ using this construction.

\section{Contents.}

1. Introduction.

2. Bogomolny Equations. $\quad 377$

3. Twistors. 378

4. Adapted Coordinates. 382

5. Compactness. 385

6. Extra Structure. 393

7. $\widetilde{T P^{1}}$ as a conic. $\quad 396$

8. Compact Twistor Fibration. 399

9. The Connection and Higgs' Field. 400

10. Extra structure. 403

11. Chern class equals energy. 405

12. Ward's Construction. 408

13. Example of a $\mathrm{U}(3)$ uniton. 412

14. Wood's Conjecture. 415

\footnotetext{
${ }^{1}$ Research supported by NSERC and FCAR scholarships.
} 


\section{Introduction.}

Unitons are harmonic maps $S: \mathbb{S}^{2} \rightarrow U(N)$, that is, maps satisfying

$$
\frac{\partial}{\partial x}\left(S^{-1} \frac{\partial}{\partial x} S\right)+\frac{\partial}{\partial y}\left(S^{-1} \frac{\partial}{\partial y} S\right)=0
$$

More generally, harmonic maps between Riemannian manifolds $M$ and $N$ are critical values of an energy functional

$$
\operatorname{energy}(\phi: M \rightarrow N)=\frac{1}{2} \int_{M}|d \phi|^{2} .
$$

In the case of maps into a matrix group, with the standard (left-invariant) metric, the energy takes the form

$$
\operatorname{energy}(S)=\frac{1}{2} \int_{\mathbb{R}^{2}}\left(\left|S^{-1} \frac{\partial}{\partial x} S\right|^{2}+\left|S^{-1} \frac{\partial}{\partial y} S\right|^{2}\right) d x \wedge d y .
$$

The uniton equations are the corresponding Euler-Lagrange equations.

From [16, Theorem 3.6], we know that harmonic maps from $\mathbb{R}^{2} \rightarrow$ $U(N)$ extend to $\mathbb{S}^{2}$ iff they have finite energy, and that such maps are always smooth. In the following, we will use this fact and work in terms of coordinates $x$ and $y$ on $\mathbb{R}^{2}$.

Unitons are determined by $A=A_{x} d x+A_{y} d y\left(=A_{z} d z+A_{\bar{z}} d \bar{z}\right.$ in characteristic coordinates)

$$
A_{x} \stackrel{\text { def }}{=} \frac{1}{2} S^{-1} \frac{\partial}{\partial x} S, \quad A_{y} \stackrel{\text { def }}{=} \frac{1}{2} S^{-1} \frac{\partial}{\partial y} S,
$$

and a choice of initial condition, $S(\infty) \in U(N)$, as we can see by thinking of $d+2 A$ as a flat connection, and $S$ as a gauge transformation. (The choice of initial condition or basing condition gives a covariant constant basis of the bundle.) We thus have a decomposition of the $U(N)$-unitons $\operatorname{Harm}\left(\mathbb{S}^{2}, U(N)\right)$ :

$$
\operatorname{Harm}\left(\mathbb{S}^{2}, U(N)\right)=U(N) \times \operatorname{Harm}^{*}\left(\mathbb{S}^{2}, U(N)\right),
$$

where

$$
\operatorname{Harm}^{*}\left(\mathbb{S}^{2}, U(N)\right) \stackrel{\text { def }}{=}\left\{S \in \operatorname{Harm}\left(\mathbb{S}^{2}, U(N)\right): S(\infty)=1\right\}
$$

will be called the based unitons. Of course, the energy doesn't depend on the basing condition, and we can write it in terms of $A$ as

$$
\text { energy }=-4 i \int \operatorname{tr} A_{z} A_{\bar{z}} d z d \bar{z} \text {. }
$$


Since $\operatorname{Harm}^{*}\left(\mathbb{S}^{2}, U(N)\right)=\left\{A=d S: S \in \operatorname{Harm}\left(\mathbb{S}^{2}, U(N)\right)\right\}$, it is useful to have equations for $A$ as well. Two u $(N)$-valued maps $A_{x}, A_{y}$ come from a map $S: \mathbb{R}^{2} \rightarrow U(N)$ in this way iff $d+2 A$ has zero curvature ( $S$ is the flat gauge) iff

$$
\begin{aligned}
0 & =d(2 A)+[2 A, 2 A] \\
& =2\left\{\frac{\partial}{\partial x} A_{y}-\frac{\partial}{\partial y} A_{x}+2\left[A_{x}, A_{y}\right]\right\} d x \wedge d y
\end{aligned}
$$

They come from a harmonic map if in addition

$$
0=d^{*} A=\frac{\partial}{\partial x} A_{x}+\frac{\partial}{\partial y} A_{y}
$$

The map $S: \mathbb{R}^{2} \rightarrow U(N)$ extends to a smooth map $\mathbb{S}^{2} \rightarrow U(N)$ iff

$$
A_{\hat{z}} \stackrel{\text { def }}{=}-z^{2} A_{z}, \text { and } A_{\hat{\bar{z}}} \stackrel{\text { def }}{=}-\bar{z}^{2} A_{\bar{z}}
$$

are smooth at $z=\infty$, where we make use of complex coordinates $z=$ $x+i y, \hat{z}=1 / z$. In terms of complex coordinates, the uniton equations are (any two of)

$$
\begin{aligned}
\frac{\partial}{\partial \bar{z}} A_{z}-\frac{\partial}{\partial z} A_{\bar{z}}+2\left[A_{\bar{z}}, A_{z}\right] & =0 \\
\frac{\partial}{\partial \bar{z}} A_{z}+\left[A_{\bar{z}}, A_{z}\right] & =0 \\
\frac{\partial}{\partial z} A_{\bar{z}}+\left[A_{z}, A_{\bar{z}}\right] & =0 .
\end{aligned}
$$

In this paper we prove

Theorem A. The space of based unitons, $\operatorname{Harm}^{*}\left(\mathbb{S}^{2}, U(N)\right)$, is isomorphic to the space of $U(N)$-uniton bundles, with energy corresponding to the second Chern class.

where the uniton bundles are bundles on $\widetilde{T \mathbb{P}^{1}} \stackrel{\text { def }}{=} \mathbb{P}(\mathcal{O} \oplus \mathcal{O}(2))$, the fibrewise compactification of the tangent bundle $T \mathbb{P}^{1}$ of the complex projective line.

Let $(\lambda, \eta)$ and $\left(\hat{\lambda}=1 / \lambda, \hat{\eta}=\eta / \lambda^{2}\right)$ be coordinates on $T \mathbb{P}^{1} \cong \mathcal{O}_{\mathbb{P}^{1}}(2)$, where $\lambda$ is the usual coordinate on $\mathbb{P}^{1}$ and $\eta$ is the coordinate associated to $d / d \lambda$. Meromorphic sections $(s)$ of $T \mathbb{P}^{1}$ give all the holomorphic sections of 
$\widetilde{T \mathbb{P}^{1}}([\mathrm{~s}, 1]$ in projective coordinates $)$, except the section at infinity $([1,0])$. We will use the following notation for curves on $\widetilde{T P}^{1}$ :

$$
\begin{aligned}
P_{\lambda} & \left.=\pi^{-1}\left(\lambda \in \mathbb{P}^{1}\right)=\text { a pfibre (silent } \mathrm{p}\right) \\
G_{0} & =\{(\lambda,[0,1])\}=(\text { graph of }) \text { zero section of } T \mathbb{P}^{1} \\
G_{\infty} & =\{(\lambda,[1,0])\}=\text { infinity section of } \widetilde{T \mathbb{P}^{1}} \\
G_{\eta=s} & =\{(\lambda,[s(\lambda), 1])\} .
\end{aligned}
$$

If $y=(a, b, c) \in \mathbb{C}^{3}$, we will also write $G_{y}$ for $G_{\eta=\frac{1}{2}\left(a-2 b \lambda-c \lambda^{2}\right)}$.

We define two 'real structures', i.e. antiholomorphic involutions, on $T \mathbb{P}^{1}$ by

$$
\tau^{*}(\lambda, \eta)=\left(-1 / \bar{\lambda},-\bar{\lambda}^{-2} \bar{\eta}\right), \quad \sigma^{*}(\lambda, \eta)=\left(1 / \bar{\lambda},-\bar{\lambda}^{-2} \bar{\eta}\right) .
$$

On $\mathbb{C}^{3} \cong H^{0}\left(\mathbb{P}^{1}, \mathcal{O}(2)\right)$, the space of finite sections, they act by

$$
\tau^{*}(a, b, c)=(\bar{c}, \bar{b}, \bar{a}), \quad \sigma^{*}(a, b, c)=(\bar{c},-\bar{b}, \bar{a}) .
$$

Since these real structures are related by time translation

$$
\begin{aligned}
\delta_{t}:(\lambda, \eta) & \mapsto(\lambda, \eta-2 t \lambda) \\
(a, b, c) & \mapsto(a, b+t, c)
\end{aligned}
$$

(real time for one is imaginary time for the other) we need only one real structure $(\sigma)$. We use both to simplify the exposition and to maintain contact with previous results for monopole bundles which we will require.

Definition of Uniton Bundles. A rank $N$, or $U(N)$, uniton bundle, $\mathcal{V}$, is a holomorphic rank $N$ bundle on $\widetilde{T P}^{1}$ which is a) trivial when restricted to the following curves in $\widetilde{T P}^{1}$

1. the section at infinity

2. nonpolar fibres (i.e. fibres above $\lambda \in \mathbb{C}^{*} \subset \mathbb{P}^{1}$ )

3. real sections of $T \mathbb{P}^{1}$

b) is equipped with bundle lifts

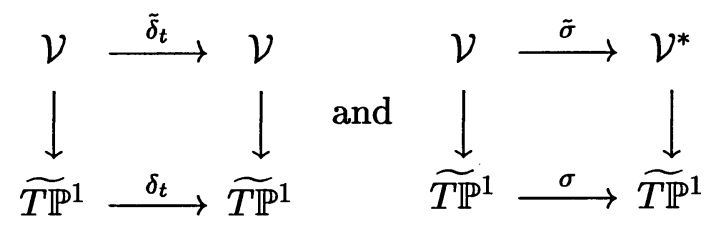


1. $\tilde{\delta}_{t}$, a one-parameter family of holomorphic transformations fixing $\mathcal{V}$ above the section at infinity, lifting $\delta_{t}$ and

2. $\tilde{\sigma}$ a norm-preserving, antiholomorphic lift of $\sigma$ such that the induced hermitian metric on $\mathcal{V}$ restricted to a fixed point of $\sigma$ is positive definite, equivalently, the induced lift to the principal bundle of frames acts on fibres of fixed points of $\sigma$ by $X \mapsto X^{*-1}$.

and c) has a framing, $\phi \in H^{0}\left(P_{-1}, \operatorname{Fr}(\mathcal{V})\right)$, of the bundle $\mathcal{V}$ restricted to the fibre $P_{-1}=\{\lambda=-1\} \subset \widetilde{T \mathbb{P}^{1}}$ such that $\tilde{\sigma}(\phi)=\phi$.

Theorem A extends a result of Ward [21] that harmonic maps $\mathbb{R}^{2} \rightarrow$ $S U(N)$ correspond to bundles on $T \mathbb{P}^{1}$ and that when $N=2$, they extend to bundles on the compactified space $\widetilde{T P}$. It also implies that the energy spectrum is discrete, as shown in [19].

The space $\widetilde{T P^{1}}$ is a rational ruled surface, and we will see from the existence of the time translation $\tilde{\delta}_{t}$ that the bundle $\mathcal{V}$ restricted to fibres, $P_{\lambda}$ of $\widetilde{T P^{1}} \rightarrow \mathbb{P}^{1}$, is trivial except for $P_{0}$ and $P_{\infty}$ which are 'jumping lines' ([10]). By proving Theorem A we can study the topology of $\operatorname{Harm}^{*}\left(\mathbb{S}^{2}, U(N)\right)$ via the spaces of allowed jumps at $P_{0}$ and $P_{\infty}$ and of monads. These are the techniques which were successful in proving the Atiyah-Jones Conjecture for Yang-Mills instantons (see [3]). Of course, this line of thinking leads to many new questions. Is the uniton number tied to the holomorphic structure of the bundle near $P_{0}$ and $P_{\infty}$ ? Is there a closed-form expression for the uniton in terms of monad data?

These questions will be the subject of a future paper. In this paper we restrict ourselves to one example and one straightforward application: a reaffirmation of Wood's conjecture that unitons are composed of rational functions of $x, y \in \mathbb{R}^{2}$ :

Corollary B. If $S: \mathbb{S}^{2} \rightarrow U(N)$ is a uniton, then the composition with $U(N) \hookrightarrow \mathrm{GL}(N)$ is rational, i.e. the functions in $x$ and $y$ which make up the matrix $S \in U(N)$ are rational.

As pointed out to the author by Martin Guest and the referee, Wood's Conjecture was affirmed by Valli [20] by showing that the lift of the harmonic map to the loop group (i.e. the extended solution) is real algebraic. Valli's use of interpolation theory is technically quite different from our treatment, and his proof applies to the more general case of pluriharmonic maps from simply-connected compact complex projective manifolds. For a discussion of the broader historical development and current motivation of this problem see [1]. 
To prove Theorem A we will extend a twistor construction of Hitchin and Ward (reviewed in $\S 3$ ). This is made possible by embedding the unitons in the solutions of the Bogomolny equations ( $\$ 2)$. Both geometric argument and Sobolev methods $(\S 5)$ are involved in extending the constructed bundle to the compactified space. Algebro-geometric methods are required to show that the inverse construction goes through $(\S \S 7-10)$. We complete the proof of Theorem A in $\S 11$ by computing the Chern-Weil integral. In $\S 12$ we relate our construction to Ward's construction, by means of which we construct a $U(3)$ uniton in $\S 13$ and affirm Wood's conjecture in $\S 14$.

\subsection{Extended Solutions.}

We will also make use of Uhlenbeck's extended solutions $E_{\lambda}$ (actually first employed in [15]), which encode the unitons as follows

Theorem $1.11[18,2.1]$. Let $\Omega \subset \mathbb{S}^{2}$ be a simply-connected neighbourhood and $A: \Omega \rightarrow T^{*}(\Omega) \otimes U(N)$. Then $2 A=S^{-1} d S$, with $S$ harmonic iff the curvature of the connection

$$
\mathcal{D}_{\lambda}=\left(\frac{\partial}{\partial \bar{z}}+(1+\lambda) A_{\bar{z}}, \frac{\partial}{\partial z}+\left(1+\lambda^{-1}\right) A_{z}\right)
$$

vanishes for all $\lambda \in \mathbb{C}^{*}$.

Theorem $1.13[18,2.2]$. If $S$ is harmonic and $S(\infty)=\mathbb{I}$, then there exists a unique covariant constant frame $E_{\lambda}: \mathbb{P}^{1} \rightarrow U(N)$ for the connection $\mathcal{D}_{\lambda}$ for $\lambda \in \mathbb{C}^{*}$ with

1. $E_{-1}=\mathbb{I}$,

2. $E_{1}=S$,

3. $E_{\lambda}(\infty)=\mathbb{I}$.

Moreover, $E_{\lambda}$ is analytic and holomorphic in $\lambda \in \mathbb{C}^{*}$.

Theorem 1.14 [18, 2.3]. Suppose $E: \mathbb{C}^{*} \times \Omega \rightarrow G$ is analytic and holomorphic in the first variable, $E_{-1} \equiv \mathbb{I}$, and the expressions

$$
\frac{E_{\lambda}^{-1} \bar{\partial} E_{\lambda}}{1+\lambda}, \quad \frac{E_{\lambda}^{-1} \partial E_{\lambda}}{1+\lambda^{-1}}
$$

are constant in $\lambda$ then $S=E_{1}$ is harmonic. 
The extended solution is the leitmotiv of this paper. It encodes the analytic properties of the uniton and without it we would be forced to use a lot more analysis in proving the compactness result of Theorem A. Moreover, the energy calculation, the construction of a U(3) uniton, and proof of Wood's conjecture use it essentially.

\section{Bogomolny Equations.}

The next important rewriting of the uniton equations was Ward's embedding of the harmonic map equations into the Bogomolny equations over $\mathbb{R}^{2,1}$ as time-independent solutions [21]. Since the geometry is simpler, we prefer to work with the Euclidean equations:

$$
\nabla \Phi=* F
$$

where $\nabla \doteq d+A$ is a connection, $\Phi$ is a Higgs' field, the curvature $F=\nabla \circ$ $\nabla=d A+A \wedge A$ and the Hodge-star is given by $* d y \wedge d t=d x, * d t \wedge d x=d y$, and $* d x \wedge d y=d t$. Assuming time independence of $\nabla$ and $\Phi$, the equations are

$$
\begin{aligned}
{\left[A_{t}, \Phi\right] } & =\frac{\partial}{\partial x} A_{y}-\frac{\partial}{\partial y} A_{x}+\left[A_{x}, A_{y}\right] \\
\frac{\partial}{\partial x} \Phi+\left[A_{x}, \Phi\right] & =\frac{\partial}{\partial y} A_{t}+\left[A_{y}, A_{t}\right] \\
\frac{\partial}{\partial y} \Phi+\left[A_{y}, \Phi\right] & =-\frac{\partial}{\partial x} A_{t}+\left[A_{t}, A_{x}\right] .
\end{aligned}
$$

Write the system (1.5) as $\nabla_{x} A_{x}=-\nabla_{y} A_{y}$ and $\nabla_{x} A_{y}=\nabla_{y} A_{x}$, and (2.1) as $\nabla_{x} A_{y}-\nabla_{y} A_{x}-\left[A_{x}, A_{y}\right]=\left[A_{t}, \Phi\right], \nabla_{x} \Phi=\nabla_{y} A_{t}$, and $\nabla_{y} \Phi=-\nabla_{x} A_{t}$. If

$$
A_{t}=-i A_{y}, \quad \Phi=i A_{x}
$$

the two systems are equivalent. It is important to note that $A_{t}$ and $\Phi$ are imaginary (i.e. in $i \mathrm{u}(N)$ ), as this will determine the real structure we will use on $T \mathbb{P}^{1}$.

We can use the freedom to change gauge to put any $t$-independent solution into the form (2.2); the new gauge, $g$, is given by solving $\frac{\partial}{\partial y} g g^{-1}=$ $i A_{t}-A_{y}, \frac{\partial}{\partial x} g g^{-1}=-i \Phi-A_{x}$, which we can do because the appropriate 
curvature component

$$
\begin{gathered}
{\left[\frac{\partial}{\partial x}+A_{x}+i \Phi, \frac{\partial}{\partial y}+A_{y}-i A_{t}\right]=\left(\frac{\partial}{\partial x} A_{y}-\frac{\partial}{\partial y} A_{x}+\left[A_{x}, A_{y}\right]-\left[A_{t}, \Phi\right]\right)} \\
+i\left(-\left(\frac{\partial}{\partial x} A_{t}+\left[A_{x}, A_{t}\right]\right)-\left(\frac{\partial}{\partial y} \Phi+\left[A_{y}, \Phi\right]\right)\right) \\
=\left(F_{x y}-\nabla_{t} \Phi\right)+i\left(F_{t x}-\nabla_{y} \Phi\right)
\end{gathered}
$$

vanishes for solutions. Thus a uniton is equivalent to a time independent solution of the Bogomolny equations. Of course, we still have to solve $S^{-1} d S=2 A$ (i.e. integrate) to get a uniton.

For future reference, we extract from the previous discussion the following

Theorem 2.3. The space of based unitons, $\operatorname{Harm}^{*}\left(\mathbb{S}^{2}, U(N)\right)$, is isomorphic to the space of $t$-independent solutions $\{(\nabla, \Phi)\}$ to the Bogomolny equations with finite energy $\int_{\mathbb{R}^{2}}\left|A_{x}\right|^{2}+\left|A_{y}\right|^{2}<\infty$ (equivalently, such that $\lim _{x+i y \rightarrow \infty} A_{z} / z^{2}$ exists), which are real in the sense that $A_{x}, A_{y} \in \mathrm{u}(N)$ in a gauge such that $A_{t}=-i A y$ and $\Phi=i A_{x}$.

\section{Twistors.}

Oriented lines in $\mathbb{R}^{3}$ are given by a direction and a displacement from the origin perpendicular to the line's direction. Collectively, they make up the space $T \mathbb{S}^{2} \cong T \mathbb{P}^{1}=\mathcal{O}_{\mathbb{P}^{1}}(2)$ (the exact correspondence depending on an isomorphism of $\mathbb{S}^{2}$ and $\mathbb{P}^{1}$ to be specified using stereographic projection). Reversing the direction of geodesics corresponds to the antiholomorphic involution, $\tau$, of $T \mathbb{P}^{1}$ which is the negative of the map on $T \mathbb{P}^{1}$ induced by the antipodal map, $\tau^{*} \lambda=-1 / \bar{\lambda}$, on $\mathbb{P}^{1}$. The holomorphic bundle $T \mathbb{P}^{1}$ has sections $\eta=a+b \lambda+c \lambda^{2}$, where $\lambda$ and $\eta$ are base and fibre coordinates over $\mathbb{P}^{1} \backslash\{\infty\}$. So its section space is $\mathbb{C}^{3}$.

Let $\underline{\mathbb{R}}$ be the trivial real line bundle. The geometry can be represented by a twistor fibration:

$$
\mathbb{S}^{2} \times \mathbb{R}^{3} \cong T \mathbb{P}^{1} \oplus \underline{\mathbb{R}}
$$

$\begin{array}{lll}\pi_{\mathbb{R}^{3}} \swarrow & \searrow & \pi_{T \mathbb{P}^{1}} \\ \mathbb{R}^{3} & & T \mathbb{P}^{1} .\end{array}$


The explicit twistor correspondence, associating points of $T \mathbb{P}^{1}$ to null planes in $\mathbb{C}^{3}$ and points of $\mathbb{C}^{3}$ to sections of $T \mathbb{P}^{1}$, is

$$
\begin{aligned}
& (\lambda, \eta) \in T \mathbb{P}^{1} \mapsto\left\{(a, b, c) \in \mathbb{C}^{3}: \eta=\frac{1}{2}\left(a-2 b \lambda-c \lambda^{2}\right)\right\}, \\
& (a, b, c) \in \mathbb{C}^{3} \mapsto\left\{(\eta, \lambda) \in T \mathbb{P}^{1}: \eta=\frac{1}{2}\left(a-2 b \lambda-c \lambda^{2}\right)\right\},
\end{aligned}
$$

where $\mathbb{R}^{3} \hookrightarrow \mathbb{C}^{3}$ as

$$
(x, y, t) \mapsto(x+i y, 2 t, x-i y) .
$$

The point of this construction is that there is a twistor correspondence between solutions $(\nabla, \Phi)$ to the Bogomolny equations on $\mathbb{R}^{3}$ and holomorphic bundles on $T \mathbb{P}^{1}$ which are trivial on real sections of $T \mathbb{P}^{1}$. This twistor construction is due to Hitchin and Ward. See [8] for details.

\subsection{The Bundle.}

Let $\mathcal{W}=\mathbb{C}^{N} \times \mathbb{R}^{3}$ be the trivial bundle over $\mathbb{R}^{3}$. Define the bundle $\mathcal{V} \rightarrow T \mathbb{P}^{1}$, over $\ell \in T \mathbb{P}^{1}$ by

$$
\mathcal{V}_{\ell}=\left\{s \in H^{0}\left(\ell, \pi_{\mathbb{R}^{3}}^{*} \mathcal{W}\right):\left(\nabla_{u}-i \Phi\right) s=0\right\}
$$

where the line $\ell \subset \mathbb{R}^{3}$ is parametrised by arclength, $u$, and $\nabla_{u}$ represents the covariant derivative corresponding to $\frac{\partial}{\partial u}$.

The bundle $\mathcal{V}$ comes with a natural $\bar{\partial}$-operator, i.e. an operator

$$
\left.\bar{\partial}: \Gamma\left(T \mathbb{P}^{1}, \mathcal{V} \otimes T^{(p, q)} T \mathbb{P}^{1}\right)\right) \rightarrow \Gamma\left(T \mathbb{P}^{1}, \mathcal{V} \otimes T^{(p, q+1)} T \mathbb{P}^{1}\right),
$$

which satisfies a Leibnitz rule and $\bar{\partial}^{2}=0$. This defines a complex bundle structure (since its flat sections will give local holomorphic framings).

The $\bar{\partial}$-operator is defined as follows.

1. Pull back the connection $(\mathcal{W}, \nabla)$ on $\mathbb{R}^{3}$ to $\left(\pi^{*} \mathcal{W}, \pi^{*} \nabla\right)$ by the projection $\pi: \mathbb{R}^{3} \times \mathbb{P}^{1} \rightarrow \mathbb{R}^{3}$.

2. The embedding $i: \mathbb{P}^{1} \hookrightarrow \mathbb{R}^{3}$ induces a splitting of $\mathbb{R}^{3} \times \mathbb{P}^{1} \rightarrow \mathbb{P}^{1}$ into

$$
T \mathbb{P}^{1} \oplus<\text { normal bundle }>.
$$


On the first component, $T \mathbb{P}^{1}$, we put the restriction of $\pi_{\mathbb{R}^{3}}^{*} \nabla$ on $\mathbb{R}^{3} \times \mathbb{P}^{1}$. On the normal bundle, we put $\pi_{\mathbb{R}^{3}}^{*}\left(\nabla_{u}-i \Phi\right)$.

Since $T \mathbb{P}^{1}$ has a complex structure, we can split $T_{\mathbb{C}} T \mathbb{P}^{1}$ into holomorphic and antiholomorphic parts. The restriction of the connection to the antiholomorphic part, $\bar{\nabla}$, acts on sections of $\left.\mathcal{W}\right|_{\ell}$ and since $\left\{\bar{\nabla}, \nabla_{u}-i \Phi\right\}$ are involutive (as a consequence of the Bogomolny equations) it induces a $\bar{\partial}$-operator on $\mathcal{V}$.

Theorem $3.5[13]$. If $(\nabla, \Phi)$ is a unitary solution of the Bogomolny equations $\nabla \Phi=* F$ on $\mathbb{R}^{3}$, then $\mathcal{V}$ is in a natural way a holomorphic bundle on the space of geodesics $T \mathbb{P}^{1}$ such that

1. $\mathcal{V}$ is trivial on every real ( $\tau$-invariant) section;

2. there exists a positive-definite, norm-preserving, antiholomorphic bundle map $\tilde{\tau}: \mathcal{V} \rightarrow \mathcal{V}^{*}$ lifting $\tau$.

Conversely, every such $\mathcal{V}$ defines a solution of the Bogomolny equations.

(Note that this theorem is a generalisation of [8, Theorem 4.2], which is stated in a way specific to $S U(2)$.)

We have encoded unitons as solutions $(\nabla, \Phi)$ of the Bogomolny equations with special properties (time invariance, finiteness, reality). We will show in $\S 4$ that these properties correspond to properties of the bundle $\mathcal{V} \rightarrow T \mathbb{P}^{1}$. Namely,

1. time translation induces a one-complex-parameter family of automorphisms of $T \mathbb{P}^{1}$ which lift to bundle maps.

2. Finiteness translates as an extension of the bundle to the fibrewise compactification of $T \mathbb{P}^{1}$. The time translation $\delta_{t}$ fixes the divisor at infinity and the bundle lift $\widetilde{\delta}_{t}$ is the trivial bundle map over the divisor at infinity.

3. Reality of the uniton solutions translates as a real structure (a fixed antiholomorphic principal bundle involution $\tilde{\sigma}$ over the antiholomorphic involution $\sigma$ of $T \mathbb{P}^{1}$, or a lift to a map $\mathcal{V} \rightarrow \mathcal{V}^{*}$ ) which is positive definite above a fixed point.

\subsection{Inverse Construction.}

Conversely, given such a bundle we can construct the Bogomolny solution. 
From the sections map

$$
\begin{aligned}
\mathbb{P}^{1} \times \mathbb{C}^{3} & \rightarrow \mathcal{O}(2) \cong T \mathbb{P}^{1} \\
\lambda,(a, b, c) & \mapsto \eta=\frac{1}{2} a-b \lambda-\frac{1}{2} c \lambda^{2}
\end{aligned}
$$

we get a pullback of the bundle $\mathcal{V}$ to $\mathbb{P}^{1} \times \mathbb{C}^{3}$. Over the open set $Y$ of sections over which $\mathcal{V}$ is trivial, we can push this bundle down from $\mathbb{P}^{1} \times Y$ to a trivial bundle over $Y$. Call this bundle $\mathcal{W} \rightarrow Y$.

Now put the quadratic form $(d b)^{2}+(d a)(d c)$ on the holomorphic tangent space to $\mathbb{C}^{3}$. To a complex metric we can associate null planes on which the restricted metric is degenerate, and null lines on which the restricted metric is zero. Each null line lies in a unique null plane (its orthogonal complement). The null planes are parametrised by $p \in T \mathbb{P}^{1}$ and given by the space of sections of $T \mathbb{P}^{1}$ through $p$, i.e. if $p=(\lambda, \eta)$ then $(a, b, c)$ are constrained by $\eta=\frac{1}{2}\left(a-2 b \lambda-c \lambda^{2}\right)$. Restricted to this plane, the metric $(d b)^{2}+\left(2 \lambda d b+\lambda^{2} d c\right)(d c)=(d b+\lambda d c)^{2}$ is degenerate. Each null plane $\Pi_{p}$ inherits a flat 'null' connection: Let $p \in T \mathbb{P}^{1}$ be the unique point of intersection of the family of sections $\Pi_{p}$. A fixed frame of $\left.\mathcal{V}\right|_{p}$ induces a frame of $\left.\mathcal{W}\right|_{\Pi_{p}}$. Define $\nabla_{\Pi_{p}}$ to be the 'null' connection for which this frame is covariant constant.

Now fix a point $y \in Y$. Some directions (lines through $y$ in $\mathbb{C}^{3}$ ) may lie in two different null planes (they correspond to sections intersecting in two distinct points), but null lines lie in unique null planes, so we can define a holomorphic connection on the null lines without ambiguity. This connection in the null lines determines a unique connection along all directions. (See [8].)

There is a second way of defining the connection. On any line in $\mathbb{C}^{3}$, pick two points $y_{1}, y_{2}$. They correspond to two sections of $T \mathbb{P}^{1}$. Since $T \mathbb{P}^{1}$ is the total space of $\mathcal{O}_{\mathbb{P}^{1}}(2)$, they intersect in two points $p_{1}, p_{2}$ (with multiplicity). We get a double point iff the sections are tangent at a point iff the line was null. To each point $p$ is associated a connection on the corresponding hyperplane $\Pi_{p_{i}} \subset \mathbb{C}^{3}$. The original line will be the intersection of the two null planes unless it was a null line, in which case it is only contained in the intersection. Taking the average of the two null connections, we get a connection on the original line, i.e.

$$
\nabla_{\text {line containing } y_{1}, y_{2}}=\frac{1}{2}\left(\nabla_{\Pi\left(p_{1}\right)}+\nabla_{\Pi\left(p_{2}\right)}\right) .
$$

In the case of a null line, we get back the null connection, because any two sections will intersect in a double point. 
Since this construction also gives a holomorphic connection, which agrees with the first on null lines, it follows from the preceding discussion that they are identical.

\subsection{Higgs' Field.}

As we defined $\nabla$ along a line as $1 / 2\left(\nabla_{\Pi}+\nabla_{\tau \Pi}\right)$, where the line is the intersection of a null plane and its conjugate, we can also define

$$
\Phi d \chi=\frac{i}{2}\left(\nabla_{\Pi}-\nabla_{\tau \Pi}\right)
$$

where the coordinate $\chi$ on the line of intersection can be well defined. (See [9].)

In $\S \S 7-10$, we will use complex algebraic methods to understand what happens in the finite (energy) case. The space of lines, $T \mathbb{P}^{1}$, can be embedded into $\mathbb{P}^{3}$ as a quasi-projective variety. It can be compactified by adding a singular point. Since $\mathcal{V}$ is trivial over the section at infinity, we get a bundle over this variety (which is a degenerate conic). Sections of $T \mathbb{P}^{1}$ correspond to certain hyperplane sections. In fact, the hyperplane sections of $\mathbb{P}^{3}$ are given by $\left(\mathbb{P}^{3}\right)^{*}$, and the sections of $T \mathbb{P}^{1} \rightarrow \mathbb{P}^{1}$ correspond to the set of hyperplane sections which do not meet the singular point $\mathbb{C}^{3} \subset \mathbb{P}^{3}$. We work out that the points we need to add from $\mathbb{P}^{2}=\mathbb{P}^{3} \backslash \mathbb{C}^{3}$ are just the set of hyperplane sections restricted to which the bundle is trivial, so we do in fact get back a (finite) uniton.

\section{Adapted Coordinates.}

In this and the next sections we assume the pair $(\nabla, \Phi)$ comes from a uniton in the way specified in $\S 2$.

Left-inverse to stereographic projection is the embedding $\mathbb{P}^{1} \hookrightarrow \mathbb{R}^{3}$ :

$$
\lambda \stackrel{i}{\mapsto}\left(\frac{\lambda+\bar{\lambda}}{1+\lambda \bar{\lambda}},-i \frac{\lambda-\bar{\lambda}}{1+\lambda \bar{\lambda}}, \frac{1-\lambda \bar{\lambda}}{1+\lambda \bar{\lambda}}\right)
$$

alternatively, $\mathbb{P}^{1} \hookrightarrow \mathbb{C} \times \mathbb{R}$ :

$$
\lambda \stackrel{i}{\mapsto}\left(\frac{2 \lambda}{1+\lambda \bar{\lambda}}, \frac{1-\lambda \bar{\lambda}}{1+\lambda \bar{\lambda}}\right) .
$$

Using this inclusion we get an exact sequence of (real) bundles over $\mathbb{P}^{1}$ :

$$
\left.0 \rightarrow T \mathbb{P}^{1} \stackrel{i_{*}}{\rightarrow} T \mathbb{R}^{3}\right|_{\mathbb{P}^{1}} \cong \mathbb{P}^{1} \times \mathbb{R}^{3} \rightarrow N_{\mathbb{P}^{1}} \rightarrow 0,
$$


where $N_{\mathbb{P}^{1}}$ is the normal bundle of the embedding, and

$$
\begin{aligned}
& i_{*}\left(\frac{\partial}{\partial \lambda}\right)=\frac{2}{(1+\lambda \bar{\lambda})^{2}} \frac{\partial}{\partial z}-2 \frac{\bar{\lambda}^{2}}{(1+\lambda \bar{\lambda})^{2}} \frac{\partial}{\partial \bar{z}}-2 \frac{\bar{\lambda}}{(1+\lambda \bar{\lambda})^{2}} \frac{\partial}{\partial t} \\
& i_{*}\left(\frac{\partial}{\partial \bar{\lambda}}\right)=-2 \frac{\bar{\lambda}^{2}}{(1+\lambda \bar{\lambda})^{2}} \frac{\partial}{\partial z}+\frac{2}{(1+\lambda \bar{\lambda})^{2}} \frac{\partial}{\partial \bar{z}}-2 \frac{\lambda}{(1+\lambda \bar{\lambda})^{2}} \frac{\partial}{\partial t} .
\end{aligned}
$$

The isomorphism $\mathbb{R}^{3} \times \mathbb{P}^{1} \cong T \mathbb{P}^{1} \oplus N_{\mathbb{P}^{1}}$ suggests that we find adapted coordinates to replace $z, \bar{z}, t, \lambda$ on $\mathbb{R}^{3} \times \mathbb{P}^{1}$. The fibre coordinate of the trivial real line bundle $T \mathbb{P}^{1} \oplus N_{\mathbb{P}^{1}} \rightarrow T \mathbb{P}^{1}$ will be $u$, given by

$$
\lambda, u \in \mathbb{P}^{1} \times \mathbb{R} \mapsto(\lambda, u i(\lambda)) \in \mathbb{P}^{1} \times \mathbb{R}^{3} .
$$

On the complement, $\eta i_{*}\left(\frac{\partial}{\partial \lambda}\right)$ gives us the fibre coordinate of $T \mathbb{P}^{1} \rightarrow \mathbb{P}^{1}$. The coordinate systems for a given $\lambda$ are related linearly by

$$
\left(\begin{array}{l}
\eta \\
\bar{\eta} \\
u
\end{array}\right)=\left(\begin{array}{ccc}
1 / 2 & -\lambda^{2} / 2 & -\lambda \\
-\bar{\lambda}^{2} / 2 & 1 / 2 & -\bar{\lambda} \\
\bar{\lambda} /(1+\lambda \bar{\lambda}) & \lambda /(1+\lambda \bar{\lambda}) & (1-\lambda \bar{\lambda}) /(1+\lambda \bar{\lambda})
\end{array}\right)\left(\begin{array}{l}
z \\
\bar{z} \\
t
\end{array}\right)
$$

Remark that $\eta=\frac{1}{2}\left(z-2 \lambda t-\lambda^{2} \bar{z}\right), \bar{\eta}=\frac{1}{2}\left(\bar{z}-2 \bar{\lambda} t-\bar{\lambda}^{2} z\right)$, and restricted to the plane $\{u=0\}, z=\frac{2 \eta-2 \lambda^{2} \bar{\eta}}{(1+\lambda \bar{\lambda})^{2}}, \bar{z}=\frac{-2 \bar{\lambda}^{2} \eta+2 \bar{\eta}}{(1+\lambda \bar{\lambda})^{2}}, \eta=\frac{1}{2} \frac{(1+\lambda \bar{\lambda})\left(z+\lambda^{2} \bar{z}\right)}{1-\lambda \bar{\lambda}}, \bar{\eta}=$ $\frac{1}{2} \frac{(1+\lambda \bar{\lambda})\left(\bar{\lambda}^{2} z+\bar{z}\right)}{1-\lambda \bar{\lambda}}$ (away from $\{|\lambda|=1\}$ ). From this change of coordinates, we can relate the connection on $\mathbb{R}^{3}$ in the two coordinate systems.

Although we have not made the distinction, changing the $\mathbb{R}^{3}$ coordinates in a $\lambda$ dependent way also affects $\frac{\partial}{\partial \lambda}$. To be precise, we should have used coordinates $\lambda, \bar{\lambda}, z, \bar{z}, t$ and $\lambda^{\prime}, \bar{\lambda}^{\prime}, \eta, \bar{\eta}, u$, with $\lambda^{\prime}=\lambda$. The distinction will be important when we want to show that the $\bar{\partial}$-operator extends to $\eta \in \mathbb{P}^{1}$ in some neighbourhood of $\lambda=0$, because we will need to work with $(\eta, \bar{\eta}, u)$ coordinates.

We make use of the fact that if $\zeta$ and $\chi$ are two choices of coordinates, $d \zeta=\Lambda d \chi \Longleftrightarrow \frac{\partial}{\partial \chi}=\Lambda^{t} \frac{\partial}{\partial \zeta}$. We have calculated $\left(\begin{array}{l}\eta \\ \bar{\eta} \\ u\end{array}\right)=B\left(\begin{array}{l}z \\ \bar{z} \\ t\end{array}\right)$ above. It follows that

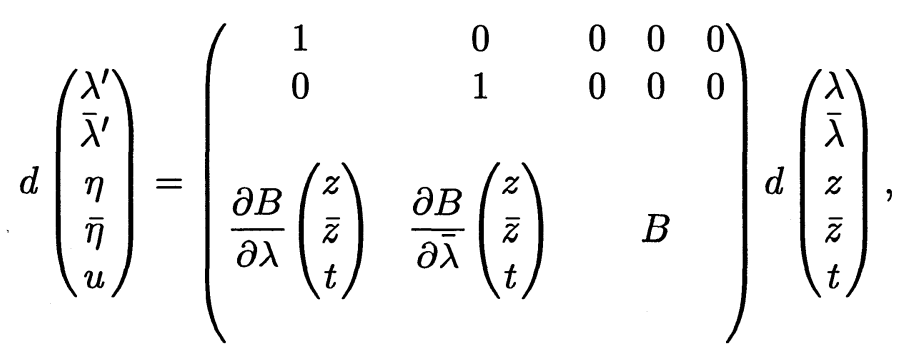


and for example

$$
\begin{aligned}
\frac{\partial}{\partial \bar{\lambda}} & =\frac{\partial}{\partial \bar{\lambda}^{\prime}}+\left(\frac{\partial B}{\partial \bar{\lambda}}\left(\begin{array}{c}
z \\
\bar{z} \\
t
\end{array}\right)\right)^{t}\left(\begin{array}{c}
\frac{\partial}{\partial \eta} \\
\frac{\partial}{\partial \bar{\eta}} \\
\frac{\partial}{\partial u}
\end{array}\right) \\
& =\frac{\partial}{\partial \bar{\lambda}^{\prime}}+\left(\frac{2 \lambda}{(1+\lambda \bar{\lambda})} \bar{\eta}-u\right) \frac{\partial}{\partial \bar{\eta}}+\frac{2}{(1+\lambda \bar{\lambda})^{2}} \eta \frac{\partial}{\partial u}
\end{aligned}
$$

So an element of the kernel of the three operators $\nabla_{u}-i \Phi, \nabla_{\bar{\eta}}, \frac{\partial}{\partial \bar{\lambda}}$ is also in the kernel of

$$
\nabla_{\bar{\lambda}^{\prime}} \stackrel{\text { def }}{=} \frac{\partial}{\partial \bar{\lambda}^{\prime}}-\left(\frac{2 \lambda}{(1+\lambda \bar{\lambda})} \bar{\eta}-u\right) A_{\bar{\eta}}-\frac{2}{(1+\lambda \bar{\lambda})^{2}} \eta\left(A_{u}-i \Phi\right) .
$$

In these coordinates, recalling (2.2)

$$
\begin{aligned}
\nabla_{u}-i \Phi & =\frac{1}{1+\lambda \bar{\lambda}}\left[2 \lambda \frac{\partial}{\partial z}+2 \bar{\lambda} \frac{\partial}{\partial \bar{z}}+(1-\lambda \bar{\lambda}) \frac{\partial}{\partial t}+2(1+\lambda)\left(A_{z}+\bar{\lambda} A_{\bar{z}}\right)\right] \\
\nabla_{\bar{\eta}} & =\frac{2}{(1+\lambda \bar{\lambda})^{2}}\left[-\lambda^{2} \frac{\partial}{\partial z}+\frac{\partial}{\partial \bar{z}}-\lambda \frac{\partial}{\partial t}+(1+\lambda)\left(-\lambda A_{z}+A_{\bar{z}}\right)\right] \\
\frac{\partial}{\partial \bar{\lambda}} & =\frac{\partial}{\partial \bar{\lambda}} \\
\nabla_{\bar{\lambda}^{\prime}} & =\frac{\partial}{\partial \bar{\lambda}^{\prime}}-\frac{2(1+\lambda)}{(1+\lambda \bar{\lambda})^{3}}\left(\left(2 \eta-2 \lambda^{2} \bar{\eta}+\lambda(1+\lambda \bar{\lambda}) u\right) A_{z}\right. \\
& \left.+(2 \bar{\lambda} \eta+2 \lambda \bar{\eta}-(1+\lambda \bar{\lambda}) u) A_{\bar{z}}\right) .
\end{aligned}
$$

Sections of the bundle $\mathcal{V}$ correspond to elements of the intersection of the kernels of these operators. Roughly speaking, the system has enough solutions if it is involutive (see [22]).

Lemma 4.4. The system

$$
\left\{\nabla_{u}-i \Phi, \nabla_{\bar{\eta}}, \frac{\partial}{\partial \bar{\lambda}}\right\}=\left\{\nabla_{u}-i \Phi, \nabla_{\bar{\eta}}, \nabla_{\bar{\lambda}^{\prime}}\right\}
$$

is involutive iff $S$ is harmonic.

Proof. A system of differential operators given by generators is involutive iff all Lie brackets of pairs of generating operators lie in the system, i.e. in 
the span of the generators. Of course any other set of generators is as good, and finding alternative generators which have vanishing Lie brackets makes things simpler. That said, remark that

$$
\begin{aligned}
\frac{1}{2}\left\{(1+\lambda \bar{\lambda}) \nabla_{\bar{\eta}}+\lambda\left(\nabla_{u}-i \Phi\right)\right\} & =\frac{\partial}{\partial \bar{z}}-\frac{1}{2} \lambda \frac{\partial}{\partial t}+(1+\lambda) A_{\bar{z}}, \text { and } \\
\frac{1}{2 \lambda}\left\{-\bar{\lambda}(1+\lambda \bar{\lambda}) \nabla_{\bar{\eta}}+\left(\nabla_{u}-i \Phi\right)\right\} & =\frac{\partial}{\partial z}+\frac{1}{2 \lambda} \frac{\partial}{\partial t}+\left(1+\lambda^{-1}\right) A_{z} .
\end{aligned}
$$

It follows that $\left[\nabla_{\bar{\eta}}, \nabla_{u}-i \Phi\right]=0$ iff $\left[\frac{\partial}{\partial \bar{z}}+(1+\lambda) A_{\bar{z}}, \frac{\partial}{\partial z}+\left(1+\lambda^{-1}\right) A_{z}\right]=0$. But since $A_{z}$ and $A_{\bar{z}}$ do not depend on $t$, this is the case iff $S$ is harmonic. That the entire system is involutive follows from the fact that (4.5) does not depend on $\bar{\lambda}$.

In fact, the parametrised system of connections $\left(\frac{\partial}{\partial \bar{z}}+(1+\lambda) A_{\bar{z}}, \frac{\partial}{\partial z}+\right.$ $\left.\left(1+\lambda^{-1}\right) A_{z}\right)$ having curvature zero can be trivialised over $\left\{(\lambda, z) \in \mathbb{C}^{*} \times \mathbb{S}^{2}\right\}$, giving Uhlenbeck's extended solutions.

\section{Compactness.}

We are interested in extending the bundles from $T \mathbb{P}^{1}$ to $\widetilde{T \mathbb{P}^{1}}$. This will follow from the finiteness (of the energy) of the uniton. The problem is that $\nabla=d+A$, which depends on $(\lambda, \eta, u) \in T \mathbb{P}^{1} \times \mathbb{R}$, does not have a limit as $\eta \rightarrow \infty$ (in the $1 / \eta$ coordinate), so the holomorphic structure must be defined differently.

We do so in two stages, first for $\{0 \neq \lambda \neq \infty\}$, the 'nonpolar' fibres of $\widetilde{T \mathbb{P}^{1}} \rightarrow \mathbb{P}^{1}$, then in neighbourhoods of the poles (i.e. $\lambda \in\{0, \infty\}$ ). The first step is motivated by the geometry of the problem and uses the extended solution, the second relies on Sobolev methods to give the existence of a continuous gauge in which the $\bar{\partial}$-operator is smooth.

If $S: \mathbb{P}^{1} \rightarrow U(N)$ is a uniton, both $S(x, y)=S(z, \bar{z})$ and $S(\hat{z}, \hat{\bar{z}})=$ $S(\hat{x}, \hat{y})$ are continuous, where $\hat{z}=1 / z$ etc. In terms of $A_{z}$ this means

$$
A_{z}=-\frac{1}{z^{2}} A_{\hat{z}}, \quad A_{\bar{z}}=-\frac{1}{\bar{z}^{2}} A_{\hat{z}},
$$

i.e. $A_{z}$ has a strong vanishing property as $z \rightarrow \infty$. Writing this out in terms of $x$ and $y$, we see that $A_{z}$ vanishes to order $2-\epsilon$ at infinity. In geometric terms, it means that the 'energy' of the connection is concentrated around the $t$-axis in $\mathbb{R}^{3}$ (see Fig. 1), so that when $\lambda \neq 0, \infty$, solutions to $\nabla_{u}-i \Phi$ should have limits as $u \rightarrow \infty$. The limit as $u \rightarrow \infty$ gives a natural 


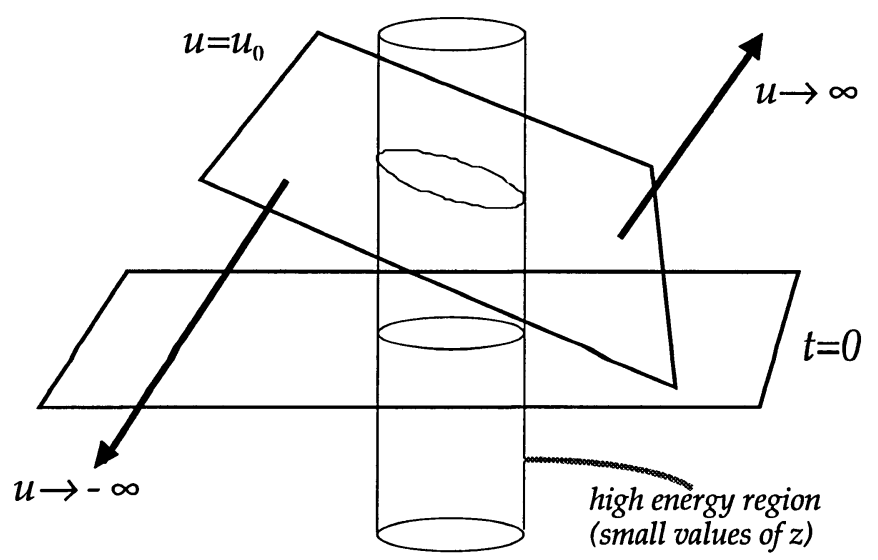

Figure 1: A picture of $\mathbb{R}^{3}$ showing the high energy cylinder and a plane $\left\{u=u_{0}\right\}$ for some direction $\lambda \in \mathbb{C}^{*},|\lambda| \neq 1$. From the picture, we would expect solutions to $\nabla_{\bar{\eta}^{\prime}}$ to extend to $\eta=\infty$, and solutions of $\nabla_{u}-i \Phi$ to extend to $u=\infty$, since in these directions the connection coefficients decrease rapidly.

holomorphic framing over 'nonpolar' fibres, $P_{\lambda}, \lambda \neq 0, \infty$, which extends to $\eta=\infty$, giving us the compactification there.

Because $\left\{\nabla_{\bar{\eta}}, \nabla_{\bar{\lambda}^{\prime}}, \nabla_{u}-i \Phi\right\}$ is involutive, solutions to this system locally correspond to solutions of $\left\{\nabla_{\bar{\eta}}, \nabla_{\bar{\lambda}^{\prime}}\right\}$ restricted to a plane $\left\{u=u_{0}\right\}$ for some $u_{0}$. As the figure suggests, away from the poles this makes sense for $u_{0}=\infty$. Near the poles, however, this doesn't work and we choose $u_{0}=0$. The transition between the resulting frames amounts to integrating $\nabla_{u}-i \Phi$ from $u=0$ to $u=\infty$.

\section{2. $G_{\infty}$ trivialisation.}

This geometric line of reasoning can be made precise analytically (see [1]), but as we said, the extended solution encodes the analytic information of the uniton, and can be exploited here. In fact, pursuing the geometric argument we have sketched uncovers the role of the extended solution. Now we restrict our attention to $\lambda \in \mathbb{C}^{*}$, i.e. to nonpolar fibres.

We saw that $\left\{\nabla_{\bar{\eta}}, \nabla_{u}-i \Phi\right\}$ has the same kernel as (4.5),

$$
\begin{aligned}
& \frac{\partial}{\partial \bar{z}}-\frac{1}{2} \lambda \frac{\partial}{\partial t}+(1+\lambda) A_{\bar{z}} \\
& \frac{\partial}{\partial z}+\frac{1}{2 \lambda} \frac{\partial}{\partial t}+\left(1+\lambda^{-1}\right) A_{z}
\end{aligned}
$$


away from the fibres $(\lambda=0, \lambda=\infty)$ where (4.5) has singularities. Both systems are 'underdetermined', since any solution can be multiplied by any holomorphic function in $\eta$ to give another solution.

The exact function, however, may be fixed by adding another differential operator to the system. Over $\left\{\lambda \in \mathbb{C}^{*}\right\}$, we can add $\frac{\partial}{\partial t}$ and get a completely integrable system on any fibre $\left\{\lambda=\lambda_{0}\right\} \subset \widetilde{T \mathbb{P}^{1}} \times \mathbb{R}, \lambda_{0} \in \mathbb{C}^{*}$, i.e. a (full) smooth connection on $\mathcal{V}$ restricted to a fibre of $\left.\widetilde{T \mathbb{P}^{1}}\right|_{\mathbb{C}^{*}} \times \mathbb{R} \rightarrow \mathbb{C}^{*}$, with zero curvature.

Since solutions of the augmented system are independent of $t$, we can push the system down to $\left\{(z, \bar{z}) \in \mathbb{R}^{2}\right\}$; i.e. all solutions to the augmented system are obtained by pulling back solutions of

$$
\left(\frac{\partial}{\partial z}+\left(1+\lambda^{-1}\right) A_{z}, \frac{\partial}{\partial \bar{z}}+(1+\lambda) A_{\bar{z}}\right)
$$

on $\mathbb{R}^{2}$ which are none other than the extended solutions.

Since the lines $\{u \in \mathbb{R}, \eta, \lambda$ constant $\}$ project to circles on $\mathbb{S}^{2}$ (Fig. 2), and the extended solution is defined on $\mathbb{S}^{2} \times \mathbb{C}^{*}$, the kernel of $\left(\nabla_{\bar{\eta}}, \nabla_{\bar{\lambda}^{\prime}}, \nabla_{u}-\right.$ $i \Phi)$ extends to

$$
\left\{(\lambda, \eta, u) \in \mathbb{C}^{*} \times \mathbb{C} \times \mathbb{S}^{1}\right\} .
$$

If we require our extended solutions to be based at $\infty$, i.e. $E_{\lambda}(\infty)=\mathbb{I}$, then the lifted solution is constant (II) at $u=\infty$, and we can take this to define the $G_{\infty}$ trivialisation of $\mathcal{V}$ restricted to nonpolar fibers of $\widetilde{T P}$.

Similarly, away from $\{|\lambda|=1\}$, the equator, $\eta$-planes

$$
\left\{\eta \in \mathbb{C}, \lambda=\lambda_{0}, u=u_{0}\right\}
$$

are mapped to $\mathbb{S}^{2} \backslash\{\infty\}$, so the lifted extended solution extends to

$$
\left\{(\lambda, \eta, u) \in \mathbb{C}^{*} \times \mathbb{P}^{1} \times \mathbb{S}^{1}:|\lambda| \neq 1\right\} .
$$

(Concentric circles $\left\{|\eta|=r, \lambda=\lambda_{0}, u=u_{0}\right\}$ are mapped to nested circles on $\mathbb{S}^{2}$ containing $\infty$.) Parallel translation by $\nabla_{u}-i \Phi$ from $\{u=\infty\}$ to $\left\{u=u_{0}\right.$, finite $\}$ will give the transition between the $G_{\infty}$ trivialisation of the nonpolar fibres and the extension of the bundle in polar neighbourhoods which we define presently. 

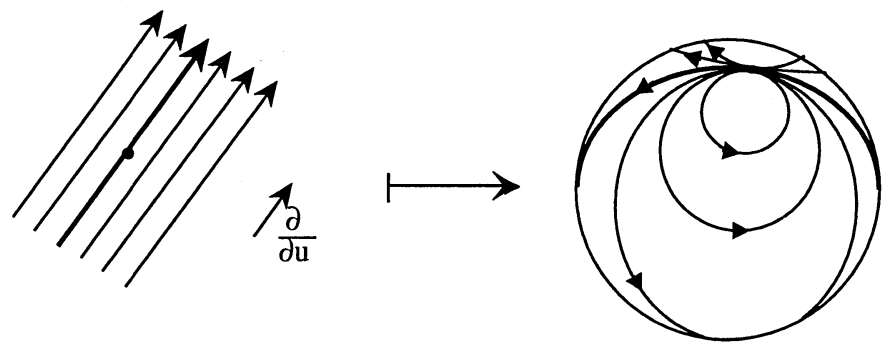

Figure 2: For a fixed value of $\lambda \in \mathbb{C}^{*}$, lines parallel to $\frac{\partial}{\partial u}$ in $\mathbb{R}^{3}$ are projected onto lines on $\mathbb{R}^{2}$ which are completed as circles on $\mathbb{S}^{2}$

\subsection{Polar neighbourhoods.}

In $\S 3$ we defined the holomorphic structure of $\mathcal{V} \mapsto \widetilde{T \mathbb{P}^{1}}$ by specifying a $\bar{\partial}$-operator. We have just seen that for $\lambda \in \mathbb{C}^{*}$, the extended solution integrates this operator to give a trivialisation of $\left.\mathcal{V}\right|_{\widetilde{T C}^{*}}$. Over 'polar' neighbourhoods ( $\lambda$ near 0 or $\infty$ ) we show that the $\bar{\partial}$-operator extends to a smooth operator near $\eta=\infty$ (equivalently $\hat{\eta}=\infty$ ), after a continuous change of gauge, showing that bundles associated to unitons extend to the fibrewise compactification-as holomorphic bundles. (A priori we don't know that the $\bar{\partial}$ is integrable near $G_{\infty}$; this follows from smoothness.) The sets

$$
\begin{aligned}
U_{0} & =\{(\lambda, \eta): \lambda \neq \infty,|\eta| \leq \infty\} \\
U_{\infty} & =\{(\lambda, \eta):|\lambda|<1 / 2, \eta \neq 0\} \\
\hat{U}_{0} & =\{(\hat{\lambda}, \hat{\eta}): \hat{\lambda} \neq \infty,|\hat{\eta}| \leq \infty\} \\
\hat{U}_{\infty} & =\{(\hat{\lambda}, \hat{\eta}):|\hat{\lambda}|<1 / 2, \hat{\eta} \neq 0\} \\
U & =\{(\lambda, \eta): 0 \neq \lambda \neq \infty\}=\mathbb{C}^{*} \times \mathbb{P}^{1},
\end{aligned}
$$

form a cover of $\widetilde{T \mathbb{P}^{1}}$. Let

$$
\Xi=\left\{\left|\bar{\eta}^{\prime}\right| \leq 1\right\}, \quad \Lambda=\{|\lambda| \leq 1\} .
$$


We will smooth the $\bar{\partial}$-operator on $U_{\infty}$. The situation on $\hat{U}_{\infty}$ is similar. On $U_{\infty}$ the $\bar{\partial}$-operator has components

$$
\begin{aligned}
\nabla_{\bar{\eta}^{\prime}} & =-\bar{\eta}^{2} \nabla_{\bar{\eta}} \\
& =\frac{\partial}{\partial \bar{\eta}^{\prime}}-\frac{(1+\lambda)(1+\lambda \bar{\lambda})^{2}}{2}\left\{\frac{\lambda}{\left(\lambda^{2}-\bar{\eta}^{\prime} / \eta^{\prime}\right)^{2}} A_{\hat{z}}-\frac{1}{\left(1-\bar{\lambda}^{2} \bar{\eta}^{\prime} / \eta^{\prime}\right)^{2}} A_{\hat{\bar{z}}}\right\} \\
& \stackrel{\text { def }}{=} \frac{\partial}{\partial \bar{\eta}^{\prime}}+B_{0} A_{\hat{z}}+B_{1} A_{\hat{\bar{z}}},
\end{aligned}
$$

$$
\nabla_{\bar{\lambda}^{\prime}}=\frac{\partial}{\partial \bar{\lambda}^{\prime}}-\frac{2(1+\lambda)}{(1+\lambda \bar{\lambda})^{3}}\left[\frac{\bar{\eta}^{\prime}}{1-\lambda^{2} \eta^{\prime} / \bar{\eta}^{\prime}} A_{\hat{z}}+\frac{\bar{\lambda} \bar{\eta}^{2} / \eta^{\prime}+\lambda \bar{\eta}^{\prime}}{\left(1-\bar{\lambda}^{2} \bar{\eta}^{\prime} / \eta^{\prime}\right)^{2}} A_{\hat{\bar{z}}}\right]
$$

where for simplicity we restrict to the hypersurface $u=0$.

Both operators are smooth away from $\eta^{\prime}=0$, and near $\eta^{\prime}=0$ are smooth functions of $\eta^{\prime}, \bar{\eta}^{\prime}$ and $\bar{\eta}^{\prime} / \eta^{\prime}$. Since $A_{\hat{z}}$ and $A_{\hat{\bar{z}}}$ are smooth near $\eta^{\prime}=0$ (for $\lambda \in \Lambda$ ), the degree of smoothness of the $\bar{\partial}$-operator is determined by that of the coefficients $B_{i}$. For example, the $\left(\bar{\eta}^{\prime} / \eta^{\prime}\right)$ factor is bounded and hence locally lies in $\left(L^{2}\right)$, but its derivative does not.

Atiyah and Bott have determined when local smoothing gauges exist in one complex dimension $([2,5.1, \S 14])$. By approaching the smoothing as a parametrised one-dimensional problem and taking advantage of the special form of the singularity, we will be able to find a continuous holomorphic gauge. Such gauges might not exist in general for $\bar{\partial}$-operators with coefficients in $L_{0}^{2}$ but without this particular singularity structure.

Because all the objects we will be dealing with, eg. $B_{i}$, are smooth away from $\eta^{\prime}=0$ and depend smoothly on $\lambda \in \Lambda$, integrability ( $L_{k}^{p}$, i.e. $L^{p}$ integrability of partial derivatives up to order $k$ ) on $\Xi \times \Lambda$, and on fibres of $\Xi \times \Lambda \rightarrow \Lambda$ are equivalent. In fact, $B_{i} \in L_{0}^{2}(\Lambda \times \Xi, \mathrm{gl}(N))$ can also be seen as a smooth map valued in a function space:

$$
\mathcal{B}_{i} \in C^{\infty}\left(\Lambda, L_{0}^{2}(\Xi, \operatorname{gl}(N))\right. \text {. }
$$

By taking the second view of $B_{i}$, as a smooth function valued in a function space, we reinterpret the search for a smooth gauge as a parametrised onecomplex-dimensional problem.

The basic tool for proving smoothness is the

Sobolev Lemma. There are inclusions

$$
\begin{array}{r}
L_{2}^{2}(\Xi, \operatorname{gl}(N)) \subset C^{0}(\Xi, \operatorname{gl}(N)) \text { and } \\
L_{3}^{2}(\Lambda \times \Xi, \operatorname{gl}(N)) \subset C^{0}(\Lambda \times \Xi, \operatorname{gl}(N))
\end{array}
$$


which are continuous with respect to the Sobolev and supremum norms respectively.

For our purposes, continuity of the inclusions will be very important. See $[6, \mathrm{p} 86]$ for a proof.

Lemma 5.9. The operator $P: g \mapsto \frac{\partial}{\partial \bar{\eta}^{\prime}} g g^{-1}$ can be extended to a smooth invertible map

$$
P: L_{k}^{2}(\Xi, G L(N, \mathbb{C}))_{0} \rightarrow L_{k-1}^{2}(\Xi, \operatorname{gl}(N, \mathbb{C}))
$$

for $k>2$, where $L_{k}^{2}()_{0}$, indicates the space of based maps, $g(0)=\mathbb{I}$.

Proof. $\quad P$ extends to a map of Sobolev spaces because

1. since $\Xi$ is compact and $L_{k}^{2}(\Xi) \subset C^{1}(\Xi)$, we can find a constant such that $\left\|g^{\prime-1}\right\|<$ const $\|g\|^{\prime}$ for $g^{\prime}$ in some neighbourhood of $g_{0}$;

2. $L_{k}^{p}\left(\mathbb{R}^{n}\right)$ is a Banach algebra for $k>n / p$ and $L_{j}^{p}$ is a topological $L_{k^{-}}^{p}$ module for $0 \leq j \leq k[2,14.5]$;

3. $\frac{\partial}{\partial \bar{\eta}^{\prime}}$ gives a map $L_{k}^{2}\left(\mathbb{C}^{n}, G L(N, \mathbb{C})\right) \rightarrow L_{k-1}^{2}\left(\mathbb{C}^{n}, \operatorname{gl}(N, \mathbb{C})\right)$ for all $k, n$.

We can calculate the derivative, $D P$, of $P$ by expanding

$$
P\left(g_{0}\left(\mathbb{I}+g_{1}\right)\right)=\frac{\partial}{\partial \bar{\eta}^{\prime}} g_{0} g_{0}^{-1}+\frac{\partial}{\partial \bar{\eta}^{\prime}} g_{1} g_{0}^{-1}-g_{1} g_{0}^{-1} \frac{\partial}{\partial \bar{\eta}^{\prime}} g_{0} g_{0}^{-1}+\phi_{g_{0}}\left(g_{1}\right),
$$

where $\phi_{g_{0}}\left(g_{1}\right)$ is tangent to the zero map (i.e. $\lim _{\left|g_{1}\right| \rightarrow 0} \frac{\phi_{g_{0}}\left(g_{1}\right)}{\left|g_{1}\right|}=0$ ),

$$
=P\left(g_{0}\right)+D P\left(g_{0}\right)\left(g_{1}\right)+\phi_{g_{0}}\left(g_{1}\right) .
$$

In particular $D P(\mathbb{I})=\frac{\partial}{\partial \bar{\eta}^{\prime}}$, so we can apply the inverse function theorem for Banach spaces [12, I.5.1] to get an inverse to $P$ in a neighbourhood of $P(\mathbb{I})=0$. It is smooth because $P$ is smooth:

We can verify the existence of higher derivatives for $P$ either by iteratively differentiating $P$, or by using the chain rule and remarking (a) that $\frac{\partial}{\partial \bar{\eta}^{\prime}}$ and $m(a, b)=a b$ are linear and multilinear respectively and hence both smooth [12, I.3.12]; and (b) that $g \mapsto g^{-1}$ is smooth because its $k^{\text {th }}$ derivative at $g=g_{0}$ is the $k$-linear map $\bigoplus^{k} L_{k}^{2}(\Xi, \operatorname{gl}(N, \mathbb{C})) \rightarrow L_{k}^{2}(\Xi, \operatorname{gl}(N, \mathbb{C}))$, given by 


$$
\left(g_{1}, \ldots, g_{k}\right) \mapsto \sum_{\sigma \in S_{k}} g_{0}^{-1} g_{\sigma(1)} g_{0}^{-1} g_{\sigma(2)} g_{0}^{-1} \cdots g_{\sigma(k)} g_{0}^{-1} .
$$

Note that we have made repeated use of (1) and (2) above.

Looking for a better gauge involves integrating $A_{\bar{\eta}^{\prime}}$, i.e. finding $C_{0}$ and $C_{1}$ such that $\frac{\partial}{\partial \bar{\eta}^{\prime}} C_{i}=-B_{i}$,

$$
\begin{aligned}
& C_{0}=\frac{(1+\lambda)(1+\lambda \bar{\lambda})^{2}}{2} \frac{-\lambda \eta^{\prime}}{\lambda^{2}-\bar{\eta}^{\prime} / \eta^{\prime}}, \\
& C_{1}=-\frac{(1+\lambda)(1+\lambda \bar{\lambda})^{2}}{2} \frac{\bar{\eta}^{\prime}}{1-\bar{\lambda}^{2} \bar{\eta}^{\prime} / \eta^{\prime}},
\end{aligned}
$$

which are not only continuous at $\eta^{\prime}=0$, but vanish there. Use

$$
g=\mathbf{1}+C_{0} A_{\hat{z}}+C_{1} A_{\hat{\bar{z}}}
$$

to change gauge

$$
A_{\bar{\eta}^{\prime}}^{g} \stackrel{\text { def }}{=} g^{-1} A_{\bar{\eta}^{\prime}} g+g^{-1} \frac{\partial}{\partial \bar{\eta}^{\prime}} g
$$

giving $A_{\bar{\eta}^{\prime}}^{g}$ the same continuity properties :

$$
\begin{aligned}
A_{\bar{\eta}^{\prime}}^{g}=\left(B_{0} A_{\hat{z}}+B_{1} A_{\hat{\bar{z}}}\right)^{g}= & g^{-1}\left(B_{0} A_{\hat{z}}+B_{1} A_{\hat{\bar{z}}}\right) g+g^{-1} \frac{\partial}{\partial \bar{\eta}^{\prime}} g \\
= & B_{0} A_{\hat{z}}+B_{1} A_{\hat{\bar{z}}}+C_{1} B_{0}\left[A_{\hat{z}}, A_{\hat{\bar{z}}}\right]+C_{0} B_{1}\left[A_{\hat{\bar{z}}}, A_{\hat{z}}\right] \\
& -\left(C_{0} A_{\hat{z}}+C_{1} A_{\hat{\bar{z}}}\right)\left(B_{0} A_{\hat{z}}+B_{1} A_{\hat{\bar{z}}}\right)\left(C_{0} A_{\hat{z}}+C_{1} A_{\hat{\bar{z}}}\right) \\
- & B_{0} A_{\hat{z}}-B_{1} A_{\hat{\bar{z}}}+C_{0}\left(B_{2} \frac{\partial}{\partial \hat{z}} A_{\hat{z}}+B_{3} \frac{\partial}{\partial \hat{\bar{z}}} A_{\hat{z}}\right) \\
+ & C_{1}\left(B_{2} \frac{\partial}{\partial \hat{z}} A_{\hat{\bar{z}}}+B_{3} \frac{\partial}{\partial \hat{\bar{z}}} A_{\hat{\bar{z}}}\right)-\left(C_{0} A_{\hat{z}}+C_{1} A_{\hat{\bar{z}}}\right) \\
& {\left[-B_{0} A_{\hat{z}}-B_{1} A_{\hat{\bar{z}}}+C_{0}\left(B_{2} \frac{\partial}{\partial \hat{z}} A_{\hat{z}}+B_{3} \frac{\partial}{\partial \hat{\bar{z}}} A_{\hat{z}}\right)\right.} \\
& \left.+C_{1}\left(B_{2} \frac{\partial}{\partial \hat{z}} A_{\hat{\bar{z}}}+B_{3} \frac{\partial}{\partial \hat{\bar{z}}} A_{\hat{\bar{z}}}\right)\right]
\end{aligned}
$$

where the terms $B_{2}, B_{3}$ arise because $\frac{\partial}{\partial \bar{\eta}^{\prime}} \neq \frac{\partial}{\partial \bar{z}}:($ on $u=0$ )

$$
\begin{aligned}
& \frac{\partial}{\partial \bar{\eta}^{\prime}}=-\bar{\eta}^{2} \frac{\partial}{\partial \bar{\eta}} \equiv-\frac{\lambda^{2}(1+\lambda \bar{\lambda})^{2}}{2\left(\lambda^{2}-\bar{\eta}^{\prime} / \eta^{\prime}\right)^{2}} \frac{\partial}{\partial \hat{z}}+\frac{(1+\lambda \bar{\lambda})^{2}}{2\left(1-\bar{\lambda}^{2} \bar{\eta}^{\prime} / \eta^{\prime}\right)^{2}} \frac{\partial}{\partial \overline{\bar{z}}} \quad\left(\bmod \frac{\partial}{\partial t}\right) \\
& \stackrel{\text { def }}{=} B_{2} \frac{\partial}{\partial \hat{z}}+B_{3} \frac{\partial}{\partial \hat{\bar{z}}} \quad\left(\bmod \frac{\partial}{\partial t}\right) .
\end{aligned}
$$


We can ignore the $\frac{\partial}{\partial t}$ terms because the connection coefficients are independent of $t$.

Since each (persistant) term contains a factor $C_{j}, A_{\bar{\eta}^{\prime}}^{g}$ vanishes on $\eta^{\prime}=0$, and is continuous. Because $\frac{\partial}{\partial \bar{\eta}^{\prime}} C_{j}=B_{j}, \frac{\partial}{\partial \bar{\eta}^{\prime}} A_{\bar{\eta}^{\prime}}^{g}$ is bounded but discontinuous, we see that $A_{\bar{\eta}^{\prime}}^{g} \in L_{1}^{2}\left(U_{\infty}, \operatorname{gl}(N, \mathbb{C})\right)$, in fact

Lemma 5.11. The map

$$
\mathcal{A}_{\bar{\eta}^{\prime}}^{g}: \Lambda \rightarrow L_{1}^{2}(\Xi, \operatorname{gl}(N, \mathbb{C}))
$$

such that

$$
\mathcal{A}_{\bar{\eta}^{\prime}}^{g}(\lambda)\left(\eta^{\prime}{ }_{0}\right)=A_{\bar{\eta}^{\prime}}^{g}\left(\lambda, \eta_{0}^{\prime}\right)
$$

is smooth.

Proof. We have to show (1) that the function $\left.A_{\bar{\eta}^{\prime}}^{g}\right|_{\lambda=\lambda_{0}}$ and its first $\eta^{\prime}, \bar{\eta}^{\prime}$ derivatives are square integrable, for all $\lambda_{0} \in \Lambda$, and (2) that $\frac{\partial}{\partial \lambda}^{p} \frac{\partial}{\partial \lambda}^{q} \mathcal{A}_{\bar{\eta}^{\prime}}^{g}$ exist and are in $L_{1}^{2}$ for all $p$ and $q$.

(1) Since

$$
A_{\bar{\eta}^{\prime}}^{g}=g^{-1} A_{\bar{\eta}^{\prime}} g+g^{-1} \frac{\partial}{\partial \bar{\eta}^{\prime}} g
$$

is smooth away from $\eta^{\prime}=0$, and has a singularity of type $\eta^{\prime} \phi\left(\eta^{\prime} / \bar{\eta}^{\prime}\right)$ there, its first derivatives in $\eta^{\prime}$ or $\bar{\eta}^{\prime}$ may have a bounded discontinuity, which doesn't affect the finiteness of the $L^{2}$ norm. In fact, we can multiply $A_{\bar{\eta}^{\prime}}^{g}$ by the complement of a bump function of arbitrarily small mass concentrated at $\eta^{\prime}=0$ and find that the map $\mathcal{A}_{\bar{\eta}^{\prime}}^{g}: \Lambda \rightarrow L_{1}^{2}(\Xi, \operatorname{gl}(N, \mathbb{C}))$ is continuous.

(2) Since $A_{\bar{\eta}^{\prime}}^{g}$ is smooth on $\left\{\eta^{\prime} \neq 0\right\}$ we can take the $\lambda$ derivatives of $\mathcal{A}_{\bar{\eta}^{\prime}}^{g}$ pointwise, i.e. when $\eta^{\prime} \neq 0$

$$
{\frac{\partial}{\partial \lambda^{\prime}}}^{p}{\frac{\partial}{\partial \bar{\lambda}^{\prime}}}^{q} \mathcal{A}_{\bar{\eta}^{\prime}}^{g}\left(\lambda_{0}\right)\left(\eta^{\prime}{ }_{0}\right)=\left.{\frac{\partial}{\partial \lambda^{\prime}}}^{p}{\frac{\partial}{\partial \bar{\lambda}^{\prime}}}^{q} A_{\bar{\eta}^{\prime}}^{g}\right|_{\left(\bar{\lambda}_{0}, \eta^{\prime}{ }_{0}\right)}
$$

Examining the terms of $A_{\bar{\eta}^{\prime}}^{g}$ one at a time, we find that all partials are continuous. For example,

$$
\begin{aligned}
\frac{\partial}{\partial \lambda^{\prime}} C_{0}= & \frac{(1+\lambda \bar{\lambda})^{2}+2 \bar{\lambda}(1+\lambda)(1+\lambda \bar{\lambda})}{2} \frac{-\lambda \eta^{\prime}}{\lambda^{2}-\bar{\eta}^{\prime} / \eta^{\prime}} \\
& +\frac{(1+\lambda)(1+\lambda \bar{\lambda})^{2}}{2} \frac{-\eta^{\prime}\left(\lambda^{2}-\bar{\eta}^{\prime} / \eta^{\prime}\right)+2 \lambda^{2} \eta^{\prime}}{\left(\lambda^{2}-\bar{\eta}^{\prime} / \eta^{\prime}\right)^{2}} \\
\frac{\partial}{\partial \lambda^{\prime}} C_{1}= & \frac{(1+\lambda \bar{\lambda})^{2}+2 \bar{\lambda}(1+\lambda)(1+\lambda \bar{\lambda})}{2} \frac{\eta^{\prime}}{\bar{\lambda}^{2}\left(1-\bar{\lambda}^{2} \bar{\eta}^{\prime} / \eta^{\prime}\right)} .
\end{aligned}
$$


Since all its partials exist and are continuous, $\mathcal{A}_{\bar{\eta}^{\prime}}^{g}: \Lambda \rightarrow L_{1}^{2}(\Xi, \operatorname{gl}(N, \mathbb{C}))$ is smooth.

Now we can exploit the fact that $P$ has a smooth inverse. As a result,

$$
\tilde{g}=P^{-1} \circ \mathcal{A}_{\bar{\eta}^{\prime}}^{g}: \Lambda \rightarrow L_{2}^{2}(\Xi, G L(N, \mathbb{C}))
$$

is a smooth map and $P(\tilde{g})=A_{\bar{\eta}^{\prime}}^{g}$. Composing with the continuous Sobolev embedding $L_{2}^{2} \hookrightarrow C^{0}$, we see that $\tilde{g}$ is a continuous change of gauge over $U_{\infty}$, such that the $\bar{\eta}^{\prime}$-operator in this gauge is trivial, i.e. $A_{\bar{\eta}^{\prime}}^{g \tilde{g}}=0$, and $A_{\bar{\lambda}}^{g \tilde{g}}=\tilde{g}^{-1} A_{\bar{\lambda}}^{g} \tilde{g}+\tilde{g}^{-1} \frac{\partial}{\partial \bar{\lambda}} \tilde{g}$ is continuous (all its ingredients are).

Since $0=\left[\nabla_{\bar{\lambda}}, \nabla_{\bar{\eta}^{\prime}}\right]=\left[\frac{\partial}{\partial \bar{\lambda}}+A_{\bar{\lambda}}^{g \tilde{g}}, \frac{\partial}{\partial \bar{\eta}^{\prime}}\right]$, it follows that $A_{\bar{\lambda}}^{g \tilde{g}}$ is meromorphic in $\eta^{\prime}$. Since it is continuous, it is holomorphic. Using the fact that $A_{\bar{\lambda}}^{g \tilde{g}}$ is smooth near $\left\{\left|\eta^{\prime}\right|=1\right\}$ and differentiating the Cauchy integral

$$
\frac{\partial^{j}}{\partial \eta^{\prime}} \frac{\partial^{k}}{\partial \lambda} \frac{\partial}{\partial \bar{\lambda}}^{l} A_{\bar{\lambda}}^{g \tilde{g}}\left(\lambda_{0}, \eta_{0}^{\prime}\right)=\int_{\left|\eta^{\prime}\right|=1} \frac{\left(\left.\frac{\partial}{\partial \lambda}^{k} \frac{\partial}{\partial \lambda}^{l} A_{\bar{\lambda}}^{g \tilde{g}}\left(\lambda, \eta^{\prime}\right)\right|_{\lambda=\lambda_{0}}\right.}{\left(\eta^{\prime}-\eta^{\prime}{ }_{0}\right)^{j}}
$$

we see that $A_{\bar{\lambda}}^{g \tilde{g}}$ is smooth on $\left\{\left|\eta^{\prime}\right|<1\right\}$. We can then find a smooth change of gauge $\hat{g}$ such that $A_{\bar{\lambda}}^{g \tilde{g} \hat{g}}=0=A_{\bar{\eta}^{\prime}}^{g \tilde{g} \hat{g}}$. Then, $s_{\infty} \stackrel{\text { def }}{=} g \tilde{g} \hat{g}$ is the required holomorphic trivialisation over $U_{\infty}$.

A similar construction works over the south pole, completing the proof that bundles arising from unitons extend to $\widehat{T \mathbb{P}^{1}}$.

\section{Extra Structure.}

\subsection{Triviality over the section at infinity.}

Lifting a based extended solution to the total space $\left(\mathbb{R}^{3} \times \mathbb{P}^{1}\right)$ gives a section $\tilde{f}$, of $\left.\mathcal{W}\right|_{\mathbb{R}^{3} \times \mathbb{C}^{*}}$, which pushes down to a choice of gauge on $\left.\mathcal{V}\right|_{\widetilde{T C}^{*}}$. Let $f_{\infty}$ and $f_{\widehat{\infty}}$ be holomorphic gauges of $\mathcal{V}$ over $U_{\infty}$ and $\hat{U}_{\infty}$ respectively (5.3), and let $\tilde{f}_{\infty}$ and $\tilde{f}_{\widehat{\infty}}$ be their pullbacks to corresponding regions of the total space. Since $\lim _{\eta \mapsto \infty} \nabla_{\bar{\lambda}^{\prime}}=\frac{\partial}{\partial \bar{\lambda}^{\prime}}$ on every plane $\{u=$ constant $\},\left.\tilde{f}_{\infty}\right|_{\left\{\eta^{\prime}=0\right\}}$ and $\left.\tilde{f}_{\widehat{\infty}}\right|_{\left\{\hat{\eta}^{\prime}=0\right\}}$ are holomorphic functions in $\lambda$. Without loss of generality we can assume $\left.\left.\tilde{f}_{\infty}\right|_{\left\{\eta^{\prime}=0\right\}} \equiv \mathbb{I} \equiv \tilde{f}_{\widehat{\infty}}\right|_{\left\{\hat{\eta}^{\prime}=0\right\}}$. In terms of the chosen gauges, $\mathcal{V}$ has transition matrices $\tilde{f}^{-1} \tilde{f}_{\infty}$ and $\tilde{f}_{\tilde{\infty}} \tilde{f}$ (which are independent of $u$ because $\tilde{f}$, $\tilde{f}_{\infty}$ and $\tilde{f}_{\widehat{\infty}}$ all solve $\left.\nabla_{u}-i \Phi\right)$. To calculate $\left.\tilde{f}\right|_{\{\eta=\infty\}}$, we appeal to the map $\mathbb{R}^{3} \times \mathbb{C}^{*} \mapsto \mathbb{S}^{2} \times \mathbb{C}^{*}$. See Fig. 2 . 
For $\lambda_{0} \in \mathbb{C}^{*}$, we saw that the lines $\left\{\eta=\eta_{0}, \lambda=\lambda_{0}\right\}$ (i.e. $u \in \mathbb{R}$ ) are mapped to circles on $\mathbb{S}^{2}$ through $\infty$, with $\infty$ missing. Similarly, for $\left|\lambda_{0}\right| \neq 1$, the planes $\left\{u=u_{0}, \lambda=\lambda_{0}\right\}$ are mapped to $\mathbb{S}^{2} \backslash\{\infty\}$. Since $\tilde{f}$ was pulled back from the based extended solution $E_{\lambda}$ on $S^{2} \times \mathbb{C}^{*}$ with $E_{\lambda}(\infty)=\mathbb{I}$, $\lim _{\eta \mapsto \infty} \tilde{f}=\mathbb{I}$ (away from $|\lambda|=1$ ). Hence $\left.\mathcal{V}\right|_{G_{\infty}}$ has constant transition matrices in these gauges and must be trivial.

\subsection{Time Invariance.}

Time translation $\left(z_{0}, t_{0}\right) \mapsto\left(z_{0}, t_{0}+t\right)$ induces a one-parameter group of transformations of $T \mathbb{P}^{1}$. In coordinates, $(\lambda, \eta) \mapsto(\lambda, \eta-t \lambda)$. The coefficients of $A_{z}, A_{\bar{z}}$ and hence $(\nabla, \Phi)$ are independent of $t$, i.e. they are invariant under the group of translations of $t$. So the space of solutions to $\nabla_{u}-i \Phi, \nabla_{\bar{\eta}}, \nabla_{\bar{\lambda}^{\prime}}$ is invariant under time translation.

On $T \mathbb{P}^{1}$, the space of oriented geodesics in $\mathbb{R}^{3}$, time translation acts by $(\lambda, \eta) \stackrel{\delta_{b}}{\mapsto}(\lambda, \eta-t \lambda)$. The geodesic itself is shifted with respect to the geodesic parameter $u$,

$$
u \stackrel{\delta_{b}}{\mapsto} u+\frac{1-\lambda \bar{\lambda}}{1+\lambda \bar{\lambda}} t
$$

So a solution $\tilde{s}(\lambda, \eta, u)$ such that $\left(\nabla_{u}-i \Phi\right) \tilde{s}=0=\nabla_{\bar{\eta}} \tilde{s}=\nabla_{\bar{\lambda}^{\prime}} \tilde{s}$ generates a family of solutions

$$
\tilde{s}_{t}(\lambda, \eta, u)=\tilde{s}\left(\lambda, \eta+\lambda t, u-\frac{1-\lambda \bar{\lambda}}{1+\lambda \bar{\lambda}} t\right) .
$$

And the map $\tilde{\delta}_{t}: \tilde{s} \mapsto \tilde{s}_{t}$, is a bundle isomorphism lifting $\delta_{t}$.

Since

$$
\lim _{z \rightarrow \infty} A=0=\lim _{z \rightarrow \infty} \Phi
$$

the bundle map is just the identity over the section at infinity. One can also see this by remarking that the $G_{\infty}$ trivialisation is preserved by the time translation map since its lift to $\mathbb{R}^{3} \times \mathbb{C}^{*}$ is independent of $t$. We will see that the specification of this map encodes the time independence of the uniton.

\subsection{The Real Structure.}

As remarked in $\S 5$, in adapting Hitchin's construction, there is some choice as to the real structure. On $\mathbb{C}^{3}$ one thinks of the real structure literally as a real slice: a three dimensional subspace of the real six dimensional $\mathbb{C}^{3}$ which as a set spans the complex three dimensional $\mathbb{C}^{3}$. Any such set 
is the fixed set of an antilinear involution - the real structure. The $\mathbb{R}^{3}$ of Hitchin's original construction is the standard real slice of standard $\mathbb{C}^{3}$ (with conjugation as the real structure). Conjugation, however, is not the appropriate real structure for our purposes. But $\mathbb{R}^{3}$ is still an invariant set of the appropriate real structure:

$$
\sigma^{*}(x, y, t)=\left(\bar{x}, \bar{y},-\bar{t}=i^{-1} \overline{i t}\right) .
$$

So when our real structure acts on $\mathcal{V} \rightarrow \mathbb{R}^{3}$ it not only conjugates the fibres, but reflects $\mathbb{R}^{3}$ in the $x-y$ plane.

One way to understand how the real structure on $\mathcal{V}$ arises is to work with frames rather than sections. This is because the real structure on $\mathcal{V}$ comes from the real structure on the complex group (GL $(N)$ in our case), which induces a real structure on the trivial principal bundle of frames of $\mathcal{W}$ over $\mathbb{R}^{3}$. The real structure fixes a real subgroup, and is $X \mapsto\left(X^{*}\right)^{-1}$ in the case of $U(N)$, which is both an involution and antiholomorphic with respect to the natural complex structure of $\mathrm{GL}(N)$. A frame of $\mathcal{V}$, either locally or at a point, is an invertible solution, $\tilde{f}$, to

$$
\left(\nabla_{a \frac{\partial}{\partial x}+b \frac{\partial}{\partial y}+c \frac{\partial}{\partial t}}-i \Phi\right) \tilde{f}=0 .
$$

Everything here lives in $\operatorname{gl}(N)$, so we can apply the transformation

$$
X \mapsto-\overline{\tilde{f}}^{-1} X \tilde{f}^{-1} t
$$

to (6.4) to get a new equation, which since $i A_{t}, i \Phi, A_{x}, A_{y} \in \mathrm{u}(N)$, gives

$$
\left(a \frac{\partial}{\partial x}+b \frac{\partial}{\partial y}+c \frac{\partial}{\partial t}+a A_{x}+b A_{y}-c A_{t}-i \Phi\right)\left(\tilde{f}^{*}\right)^{-1}=0 .
$$

Pulling back by $\sigma$, and using the fact that $A_{x}, A_{y}, A_{t}, \Phi$ are independent of $t$, we get

$$
\left(\nabla_{a \frac{\partial}{\partial x}+b \frac{\partial}{\partial y}-c \frac{\partial}{\partial t}}-i \Phi\right)\left(\sigma^{*} \tilde{f}\right)^{*-1}=0 .
$$

So the real structure on the principal bundle $\mathbb{R}^{3} \times \mathrm{GL}(N)$ induces an antiholomorphic involution of the principal bundle of frames of $\mathcal{V} \rightarrow T \mathbb{P}^{1}$ which covers $\sigma$ and which conjugates the natural frames above real sections (i.e. (frame) $\mapsto(\text { frame })^{*-1}$ in a unitary frame). The specific form of this conjugation is important-other conjugations correspond to different real groups, i.e. $\mathrm{Gl}(N, \mathbb{R}), \mathrm{U}(n, N-n)$ etc.

On the level of vector bundles, this translates into an antiholomorphic $\operatorname{map} \mathcal{V} \rightarrow \mathcal{V}^{*}$ lifting $\sigma$. 


\subsection{Framing.}

Finally, let $p$ be any fixed point of $\sigma$ contained in $P_{-1}$. We take as the framing above this point the solution to $\left(\nabla_{u}-i \Phi\right) \phi=0$ along the line $p \subset \mathbb{R}^{3}$ with

$$
\lim _{u \rightarrow \infty} \phi=\mathbb{I} .
$$

Since $p$ is fixed by $\sigma$, the real structure $X \mapsto X^{*-1}$ takes solutions of $\nabla_{u}-i \Phi$ along $p$ to solutions, and maps our particular solution to itself. Since $\left.\mathcal{V}\right|_{P_{-1}}$ is trivial,

$$
H^{0}\left(P_{-1}, \operatorname{Fr}(\mathcal{V})\right) \stackrel{\text { eval }}{\longrightarrow} \operatorname{Fr}\left(E_{p}\right)
$$

is an isomorphism. This defines the 'unitary' framing

$$
\phi \in H^{0}\left(P_{-1}, \operatorname{Fr}(\mathcal{V})\right)
$$

of the definition.

Remark 6.6. The value of this result is that since $\widetilde{T P^{1}}$ is compact, the a priori analytic uniton bundles are algebraic (see [17]). This underlies the inverse construction to follow.

\section{7. $\widetilde{T P^{1}}$ as a conic.}

As a preliminary step to inverting the bundle construction of $\S 6$, we show that $T \mathbb{P}^{1}$ can be embedded into $\mathbb{P}^{3}$ as the nonsingular subset of a conic. Consider the conic $Q$ given in homogeneous coordinates $\alpha, \beta, \gamma, \delta$ on $\mathbb{P}^{3}$ by $\beta^{2}=-4 \alpha \gamma$. This conic has a singular point at $[0,0,0,1]$. Now consider the map $f: T \mathbb{P}^{1} \rightarrow Q$ given by

$$
\begin{aligned}
& (\lambda, \eta) \mapsto\left[1,-2 \lambda,-\lambda^{2},-2 \eta\right]=[\alpha, \beta, \gamma, \delta] \\
& (\hat{\lambda}, \hat{\eta}) \mapsto\left[-\hat{\lambda}^{2}, 2 \hat{\lambda}, 1,2 \hat{\eta}\right] .
\end{aligned}
$$

The map $f$ extends to a rational map on $\widetilde{T P^{1}}$ mapping the section at infinity to the singular point. Since the bundle is trivial over the section at infinity, when we collapse this section the bundle descends to another bundle $f_{*} \mathcal{V}$ on $Q$. More precisely,

Lemma 7.2. Pullback of bundles $\left(\mathcal{Z} \mapsto f^{*} \mathcal{Z}\right)$ from $Q$ back to $\widetilde{T P}^{1}$ is an isomorphism onto the space of bundles on $\widetilde{T \mathbb{P}^{1}}$, trivial over the section at infinity, $G_{\infty}$. 


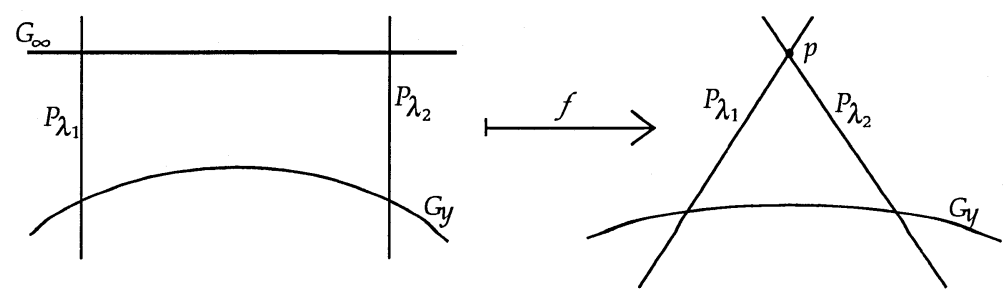

Figure 3: The embedding of $\widetilde{T \mathbb{P}^{1}} \rightarrow Q \subset \mathbb{P}^{3}$ maps the section at infinity to a singular point. Hyperplane sections of $Q$ pull back to sections, $G_{y}$, of $T \mathbb{P}^{1}$, or to unions $P_{\lambda_{1}} \cup G_{\infty} \cup P_{\lambda_{2}}$ if they contain the singular point.

Proof. Pullback of bundles is injective. (Pushforward is a left inverse of pullback.) We must show it is surjective- that every bundle trivial on $G_{\infty}$ is the pullback of a bundle on $Q$. Let $\mathcal{Z}^{\prime} \rightarrow \widetilde{T \mathbb{P}^{1}}$ be trivial on $G_{\infty}$. Away from $G_{\infty}, f$ is bijective, so $\mathcal{Z}^{\prime}$ pushes forward to a bundle on $Q$ away from the singular point. We shall use the Theorem on Formal Functions to push forward a trivialisation of $\mathcal{Z}^{\prime}$ in a neighbourhood of $G_{\infty}$ to a trivialisation of $f_{*} \mathcal{Z}^{\prime}$ in a neighbourhood of the singular point $f_{*}\left(G_{\infty}\right)$. So $f_{*} \mathcal{Z}^{\prime}$ is a bundle (a locally trivial sheaf) whose image is $\mathcal{Z}^{\prime}$, proving surjectivity.

Locally, the section at infinity, $G_{\infty}$, looks like the zero section of $\mathcal{O}_{\mathbb{P}^{1}}(-2)$. Given local coordinates $\left(\lambda, \eta^{\prime}\right)$ and $\left(\hat{\lambda}=1 / \lambda, \hat{\eta}^{\prime}=\lambda^{2} \eta^{\prime}\right)$ on $\mathcal{O}(-2)$, a transition matrix for $\mathcal{Z}^{\prime}$ is given over the intersection, $\left\{\lambda \in \mathbb{C}^{*}\right\}$, as

$$
\mathbb{I}+\eta^{\prime}\left(\phi\left(\lambda, \hat{\lambda}, \eta^{\prime}, \hat{\eta}^{\prime}\right)\right)
$$

where $\phi$ is a polynomial matrix. Since $\hat{\eta}^{\prime}=\lambda^{2} \eta^{\prime}$, we can express this in terms of two polynomials as

$$
\begin{aligned}
& \mathbb{I}+\eta^{\prime}\left(\phi^{\prime}\left(\lambda, \eta^{\prime}\right)+\phi^{\prime \prime}\left(\hat{\lambda}, \hat{\eta}^{\prime}\right)\right) \\
& \quad=\mathbb{I}+\eta^{\prime} \phi^{\prime}\left(\lambda, \eta^{\prime}\right)+\hat{\eta}^{\prime} \hat{\lambda}^{2} \phi^{\prime \prime}\left(\hat{\lambda}, \hat{\eta}^{\prime}\right),
\end{aligned}
$$

but not uniquely, as $\eta^{\prime}=\hat{\lambda}^{2} \hat{\eta}^{\prime}$ etc. We can use this property to show inductively that the bundle must be trivial on all formal neighbourhoods of $G_{\infty}$, by showing that, for any $k>0$, such a transition matrix $\in C^{1}\left(G_{\infty}^{(k)}, \operatorname{GL}(N)\right)$ is actually a coboundary, splitting as a product of holomorphic changes of gauge, i.e. that it is in the image of $C^{0}\left(G_{\infty}^{(k)}, \mathrm{GL}(N)\right)$.

A bundle is trivial on the $(k-1)$ st formal neighbourhood, $G_{\infty}^{(k-1)}$, iff its transition matrix has the form

$$
\mathbb{I}+\eta^{\prime k}(\phi)
$$


in some gauge. Using the fact that $\phi$ can be split as $\phi=\phi^{\prime}\left(\lambda, \eta^{\prime}\right)+\phi^{\prime \prime}\left(\hat{\lambda}, \hat{\eta}^{\prime}\right)$, we can make a change of gauge:

$$
\begin{aligned}
\left(\mathbb{I}-\eta^{\prime k} \phi^{\prime}\right) & \left(\mathbb{I}+\eta^{\prime k}\left(\phi^{\prime}+\phi^{\prime \prime}\right)\right)\left(\mathbb{I}-\hat{\eta}^{\prime k} \hat{\lambda}^{2 k} \phi^{\prime \prime}\right) \\
& =\mathbb{I}+\eta^{2 k}\left(\phi^{\prime} \phi^{\prime \prime}-\phi^{\prime}\left(\phi^{\prime}+\phi^{\prime \prime}\right)-\phi^{\prime \prime}\left(\phi^{\prime}+\phi^{\prime \prime}\right)\right),
\end{aligned}
$$

showing that it is trivial on $G_{\infty}^{(2 k-1)}$. Inductively, we get a trivialisation of $\mathcal{Z}^{\prime(k)}$, which is the same as a maximal rank section of $\operatorname{Hom}\left(\mathcal{E}^{\oplus N}, \mathcal{Z}^{\prime}\right)$ over the $k^{\text {th }}$ formal neighbourhood $\left(G_{\infty}^{(k)}\right)$, for $k$ arbitrarily large, where $\mathcal{E}$ is the trivial bundle. Now the Theorem on Formal Functions [7, III.11.1] says that

$$
f_{*} \operatorname{Hom}\left(\mathcal{E}^{\oplus N}, \mathcal{Z}^{\prime}\right)_{[0,0,0,1]}^{\wedge} \stackrel{\cong}{\rightarrow} \varliminf_{\longrightarrow} H^{0}\left(G_{\infty}^{(k)}, \operatorname{Hom}\left(\mathcal{E}^{\oplus N}, \mathcal{Z}^{\prime}\right)\right) .
$$

We have shown that the RHS has a maximal rank element. The LHS is the set of all sections of $\operatorname{Hom}\left(\mathcal{E}^{\oplus N}, \mathcal{Z}^{\prime}\right)$ on a neighbourhood of $f^{-1}([0,0,0,1])=$ $G_{\infty}$, up to formal equivalence, i.e. germs of sections. It must contain an element corresponding to the maximal-rank element of the RHS. That element is a section on some neighbourhood of the section at infinity which is nondegenerate on the infinity section. Since the determinant function is continuous, it must be nondegenerate on some neighbourhood where it gives a trivialisation. This trivialisation pushes forward to give a trivialisation of $f_{*} \mathcal{Z}^{\prime}$ in a neighbourhood of the singular point, so in particular $f_{*} \mathcal{Z}^{\prime}$ is a bundle.

The same construction would work with any bundle trivial over a rational curve of negative self-intersection embedded in a surface, because the splitting of $\phi$ would go through. A theorem of Castelnuovo tells us that the new surface will be smooth iff the curve has self-intersection -1 , in which case we are just blowing down.

The point of this construction is that it tells us what happens to deformed real sections in the limit (as $t \rightarrow \infty$, for example). A hyperplane in $\mathbb{P}^{3}$, $\alpha a+\beta b+\gamma c+\delta d=0$, restricted to $Q$ can be written

$$
a \alpha-2 \lambda b \alpha-\lambda^{2} \alpha c-2 \eta \alpha d=0 \quad \text { or } \quad-\hat{\lambda}^{2} \gamma a+2 \hat{\lambda} \gamma b+\gamma c z \hat{\eta} \gamma d=0 .
$$

When $d \neq 0$, we can use affine coordinates $a / d, b / d, c / d$ or just restrict to the plane $d=1$ and to pull these sections back to $T \mathbb{P}^{1}$ we restrict to the affine plane $\{\alpha=1\}$ (see (7.1)). In $T \mathbb{P}^{1}$ coordinates, the hyperplane section $(a, b, c, 1)$ is

$$
\eta=\frac{1}{2}\left(a-2 b \lambda-c \lambda^{2}\right)
$$


a holomorphic section of $T \mathbb{P}^{1} \rightarrow \mathbb{P}^{1}$. In other words the sections $\mathbb{P}^{1} \rightarrow T \mathbb{P}^{1}$ have their images cut out by hyperplane sections with $d \neq 0$.

But what about the $\mathbb{P}^{2}$ of hyperplanes with $d=0$ ? From Fig. 3, we see that these are just the hyperplanes which include the singular point. Such intersections solve $a \alpha+b \beta+c \gamma=0$ and $-4 \alpha \gamma=\beta^{2}$, so they solve $a^{2} \alpha^{2}+\left(2 a c+4 b^{2}\right) \alpha \gamma+c^{2} \gamma^{2}=0$. When $b^{2}+a c=0$ or $b=0$ the solution is a double line. In general we get two lines intersecting in the pinch point:

$$
\begin{aligned}
& a^{2} \alpha+\left(a c+2 b^{2} \pm 2 b \sqrt{a c+b^{2}}\right) \gamma=0, \\
& a^{2} b \beta=\left(-a^{2} c+a c+2 b^{2} \pm 2 b \sqrt{a c+b^{2}}\right) \gamma
\end{aligned}
$$

So the correct way to complete the set of holomorphic sections of $T \mathbb{P}^{1}$ is not by adding a section at infinity but by adding a $\mathbb{P}^{2}$ worth of closed subvarieties of $\widetilde{T \mathbb{P}^{1}}$, given by the union of the section at infinity and two fibres with multiplicity.

\section{Compact Twistor Fibration.}

Let $X \subset \mathbb{P}^{3} \times \mathbb{P}^{3 *}$ be the variety cut out by $\beta^{2}+4 \alpha \gamma=0$ and $a \alpha+$ $b \beta+c \gamma+d \delta=0$, where $a, b, c, d$ are homogeneous coordinates on the space of hyperplane sections of $\mathbb{P}^{3}, \mathbb{P}^{3} \cong \mathbb{P}^{3}$. We will use the double (twistor) fibration

$$
T \mathbb{P}^{1} \hookrightarrow Q Q^{\pi_{1} \swarrow}{ }^{X} \searrow \pi_{2} \quad \mathbb{P}^{3 *} \hookleftarrow \mathbb{C}^{3} .
$$

Pull back the bundle $\mathcal{V}$ to $X$ and push it forward to a sheaf $\mathcal{W}$ over $\mathbb{P}^{3}$. Let

$$
Y=\left\{y \in \mathbb{P}^{3^{*}}:\left.\mathcal{V}\right|_{X_{y}} \text { is trivial }\right\}
$$

and

$$
X^{\prime}=\pi_{2}^{-1}(Y)
$$

In the following, $y$ will be assumed to be in $Y$.

Grauert's Theorem ([4]) implies that $\mathcal{W} \rightarrow Y$ is locally free, i.e. a bundle, when (the sheaf of sections of) $\pi_{1}^{*} \mathcal{V}$ is flat over $Y$. Since (sections of) bundles are coherent, this follows from the flatness of $\left(X^{\prime}, \mathcal{O}_{X^{\prime}}\right) \rightarrow\left(Y, \mathcal{O}_{Y}\right)$, which is flat iff

$$
P_{y}(m)=\operatorname{dim}_{\mathbb{C}} H^{0}\left(X_{y}, \mathcal{O}_{X_{y}}(m)\right)
$$


is independent of $y \in Y$ for $m \gg 0$ ([7, III.9.2]). We can compute $P_{y}(m)$ from the long exact homology sequence associated to the embedding $X_{y} \subset$ $\mathbb{P}^{2}$ :

$$
0 \rightarrow \mathcal{O}_{\mathbb{P}^{2}}\left(-X_{y}\right) \rightarrow \mathcal{O}_{\mathbb{P}^{2}} \rightarrow \mathcal{O}_{X_{y}} \rightarrow 0 .
$$

Since $Q$ is cut out by a quadric, $X_{y}=Q \cap y$ is also a quadric in $\mathbb{P}^{2}$ (of degree two) and $\mathcal{O}_{\mathbb{P}^{2}}\left(-X_{y}\right)=\mathcal{O}_{\mathbb{P}^{2}}(-2)$. Plug this into the long exact sequence associated to (8.3)

$$
\begin{aligned}
0 & \rightarrow H^{0}\left(\mathbb{P}^{2}, \mathcal{O}_{\mathbb{P}^{2}}(m-2)\right) \rightarrow H^{0}\left(\mathbb{P}^{2}, \mathcal{O}_{\mathbb{P}^{2}}(m)\right) \rightarrow H^{0}\left(X_{y}, \mathcal{O}_{X_{y}}(m)\right) \\
& \rightarrow H^{1}\left(\mathbb{P}^{2}, \mathcal{O}_{\mathbb{P}^{2}}(m-2)\right) \rightarrow H^{1}\left(\mathbb{P}^{2}, \mathcal{O}_{\mathbb{P}^{2}}(m)\right) \rightarrow H^{1}\left(X_{y}, \mathcal{O}_{X_{y}}(m)\right) .
\end{aligned}
$$

According to Theorem $\mathrm{B}$, for some suitably large $\mu_{0}, H^{q}\left(M, \mathcal{O}\left(H^{\mu} \otimes \mathcal{V}\right)\right)=0$, for all $q>0$, and $\mu>\mu_{0}$. In particular, $H^{1}\left(\mathcal{O}_{\mathbb{P}^{2}}(m-2)\right)=0$ for $m>m_{0}$, hence

$$
h^{0}\left(\mathcal{O}_{X_{y}}(m)\right)=h^{0}\left(\mathcal{O}_{\mathbb{P}^{2}}(m)\right)-h^{0}\left(\mathcal{O}_{\mathbb{P}^{2}}(m-2)\right)
$$

which is independent of $y$.

\section{The Connection and Higgs' Field.}

The construction of the connection $\nabla$ in $\S 3$ also defines a connection on $Y$. We then have a connection over the real slice $Y_{\mathbb{R}}$ of $Y$, which is a partial compactification of $\mathbb{R}^{3}$. We show that we can push $\nabla$ down from $Y_{\mathbb{R}}$ to $\mathbb{S}^{2} \times \mathbb{R}$

\subsection{The set $Y$.}

To summarise what we know about $Y$ :

1. Finite real points are in $Y$ :

$$
\left\{[a, b, \bar{a}, 1] \in \mathbb{C}^{3} \subset \mathbb{P}^{3}: a \in \mathbb{C}, b \in \mathbb{R}\right\}=\text { fix }(\tau) \cap \mathbb{C}^{3} \subset Y
$$

since $\mathcal{V}$ is trivial on $\tau$-real sections of $T \mathbb{P}^{1}$. (In fact $t$-invariance of $\mathcal{V}$ implies fix $(\sigma) \subset\{[a, b, \bar{a}, 1]: a, b \in \mathbb{C}\} \subset Y$, as well.)

2. For infinite points, we know precisely that $Y \cap \mathbb{P}_{\text {at } \infty}^{2 *}=\left\{\right.$ hyperplane sections which contain neither $P_{0}$ nor $\left.P_{\infty}\right\}$

$$
\begin{aligned}
& \left(=\frac{\left\{\left(\lambda_{1}, \lambda_{2}\right) \in \mathbb{C}^{*} \times \mathbb{C}^{*}\right\}}{\left(\lambda_{1}, \lambda_{2}\right) \sim\left(\lambda_{2}, \lambda_{1}\right)}\right) \\
= & \left\{[a, b, c, 0] \in \mathbb{P}_{\text {at } \infty}^{2 *}: a c \neq 0\right\} \\
\cong & \mathbb{P}^{2} \backslash\left(\mathbb{P}^{1} \vee \mathbb{P}^{1}\right) .
\end{aligned}
$$


By virtue of our choice of $\mathbb{C}^{3} \subset \mathbb{P}^{3 *}$, we know that the $\mathbb{P}^{2}$ at infinity is the set of hyperplane sections of $Q$ through the singular point. Furthermore, any two such hyperplane sections either have a line in common or meet only at the singular point. From either definition of the connection, it's clear that the evaluation at the singular point gives a covariant constant frame of $\mathcal{W}$ over $Y \cap \mathbb{P}_{\text {at }}^{2 *}$. Since the standard trivialisation of $\mathcal{W}$ comes from evaluation at $P_{\lambda}$, which contains the point at $\infty$, the connection is zero along the $\mathbb{P}^{2}$ at infinity. This is exactly the property which allows us to push the connection down to $\mathbb{S}^{2}$.

\subsection{Real Points.}

We know that finite real points are in $Y$. We must calculate the infinite ones. The involution $\tau$ acts on $\mathbb{P}^{3 *}$ by $\tau(a, b, c, d)=(\bar{c}, \bar{b}, \bar{a}, \bar{d})$, so the real points of $Y \cap \mathbb{P}_{\text {at } \infty}^{2 *}$ are $\left\{[a, t, \bar{a}, 0], a \in \mathbb{C}^{*}, t \in \mathbb{R}\right\} \cong \mathbb{R} P^{2} \backslash$ pt. As the finite portion of $Y_{\mathbb{R}}$ is $\mathbb{R}^{3}$, one has

$$
Y_{\mathbb{R}} \cong \mathbb{R} P^{3} \backslash\{[\mathrm{pt}]\} .
$$

We can similarly calculate the level set

$$
\begin{array}{rlclc}
Y_{\mathbb{R}} \cap\{t=0\} & = & \mathbb{R}^{3} \cap\{t=0\} & \cup & \left(\mathbb{R} P^{2} \backslash\{[\mathrm{pt}]\}\right) \cap\{t=0\} \\
& = & \mathbb{R}^{2} & \cup & \{[a, 0, \bar{a}, 0]: a \neq 0\} \\
& = & \mathbb{R}^{2} & \cup & \mathbb{S}^{1} \\
& = & \mathbb{R} P^{2} & &
\end{array} .
$$

\subsection{Pushing down the connection.}

Let

$$
\pi: \mathbb{R} P^{2} \rightarrow \mathbb{S}^{2}
$$

be the map which maps the circle at infinity in $\mathbb{R} P^{2}$ to the infinite point in $\mathbb{S}^{2}$.

We are given a connection on the trivial bundle over $\mathbb{R} P^{2}$ with a fixed trivialisation, which restricted to the $\mathbb{S}^{1}$ at infinity is covariant constant. It is therefore plausible that the connection can be pushed down. We now check this. 
Let $[x, y, w]$ be homogenous coordiates on $\mathbb{R} P^{2}$, with $(x / w)$ and $(y / w)$ the usual affine coordinates on $\mathbb{R}^{2}$. Real projective space $\mathbb{R} P^{2}$ has two more affine coordinate patches. On each patch the connection is given by connection matrices real analytic in the affine coordinates. In terms of the patch $(x / y),(w / y)$, the condition that the given trivialisation is covariant constant on the circle at infinity translates into the fact that $A_{(x / y)}=0$ when $(w / y)=0$. Since $A$ is real analytic, we can find a second matrix such that $A_{(x / y)}=(w / y) \widetilde{A_{(x / y)}}$. This is analogous to the condition (5.1) for a connection which comes from a uniton.

Using the the relations $(x / y)=(x / w) /(y / w)$ etc., we calculate

$$
\begin{aligned}
A_{(x / w)} & =\frac{1}{(y / w)^{2}} \widetilde{A_{(x / y)}} \\
& =-\frac{(y / w)}{(x / w)^{3}} \widetilde{A_{(y / x)}}-\frac{1}{(x / w)^{2}} A_{(w / x)} \\
A_{(y / w)} & =-\frac{(x / w)}{(y / w)^{3}} \widetilde{A_{(x / y)}}-\frac{1}{(y / w)^{2}} A_{(w / y)} \\
& =\frac{1}{(x / w)^{2}} \widetilde{A_{(y / x)}} .
\end{aligned}
$$

Discarding a neighbourhood of $((x / w),(y / w))=0$ in $\mathbb{R}^{2}$, we can integrate the squared norm of the first expression for $A_{(x / w)}$ on the sectors $(y-x)(y+x)>0$ and the second expression on the other sectors. A similar argument shows that $A_{(y / w)}$ is also finite on $\mathbb{R}^{2}$. It follows that the connection on $\mathbb{R}^{2}$ comes from a finite-energy harmonic map or, equivalently, that the connection pushes down to $\mathbb{S}^{2}$.

It will follow from the proof of $t$-invariance of $(\nabla, \Phi)$ on $\mathbb{R}^{3}$ that the extension of the connection from $\mathbb{R}^{2}$ to $\mathbb{S}^{2}$ implies the extension of $(\nabla, \Phi)$ from $\mathbb{R}^{3}$ to $\mathbb{S}^{2} \times \mathbb{R}$. Specifically, we know from the discussion of the Bogomolny normalisation following (2.2) that we can put any $t$-invariant pair $(\nabla, \Phi)$ satisfying the Bogomolny equations on $\mathbb{R}^{3}$ into the form $A_{t}=-i A_{y}$, $\Phi=i A_{x}$, so that the finiteness of $A_{x}$ and $A_{y}$ at infinity certainly imply the finiteness of $A_{t}$ and $\Phi$. 


\section{Extra structure.}

\subsection{Choosing a trivialisation of $\mathcal{W} \rightarrow Y$.}

The bundle $\mathcal{V}$ is trivial over the fibre $P_{-1} \subset \widetilde{T \mathbb{P}^{1}}$ with a fixed framing $\phi$. We get a map $\mathcal{W} \rightarrow \mathbb{C}^{N}$, defined by

$$
\begin{gathered}
\mathcal{W}_{y}=H^{0}\left(G_{y}, \mathcal{V}\right) \underset{\cong}{\stackrel{\text { restr. }}{\cong}} H^{0}\left(G_{y} \cup P_{-1}, \mathcal{V}\right) \\
\stackrel{\text { restr. }}{\cong} H^{0}\left(P_{-1}, \mathcal{V}\right) \stackrel{\phi}{\cong} \mathbb{C}^{N}
\end{gathered}
$$

where we use the canonical isomorphisms coming from restriction and $G_{y}$ is a section of $T \mathbb{P} \rightarrow \mathbb{P}^{1}$. This is well defined because $y$ is either a finite point (a section of $T \mathbb{P}^{1}$ ) and intersects $P_{-1}$ in a point, or it is infinite in which case it meets the fibre at one point or on the whole fibre, in which case evaluation at any point of the fibre gives the same answer, again because $\mathcal{V}$ is trivial there. This map gives a trivialisation

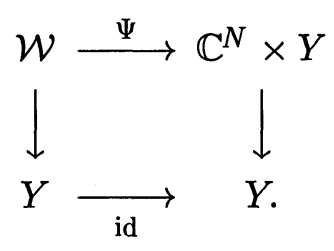

One value of this trivialisation is that in this framing the translation action $\delta_{t}$ lifts to id $\times \delta_{t}$ :

$$
\begin{aligned}
& \mathcal{W}_{\delta_{t} y}=H^{0}\left(\delta_{t} G_{y}, \mathcal{V}\right) \stackrel{\text { eval }}{\cong} \mathcal{V}_{\delta_{t} G_{y} \cap P_{-1}} \stackrel{\text { eval }}{\cong} H^{0}\left(P_{-1}, \mathcal{V}\right)=\mathbb{C}^{N} \\
& \tilde{\delta}_{t} \downarrow \quad \tilde{\delta}_{t} \downarrow \quad \downarrow \tilde{\delta}_{t}=\mathrm{id} . \\
& \mathcal{W}_{y}=H^{0}\left(G_{y}, \mathcal{V}\right) \stackrel{\text { eval }}{\cong} \mathcal{V}_{G_{y} \cap P_{-1}} \stackrel{\text { eval }}{\cong} H^{0}\left(P_{-1}, \mathcal{V}\right)=\mathbb{C}^{N}
\end{aligned}
$$

The fact that the last $\tilde{\delta}_{t}$ is the identity comes from the fact that $\tilde{\delta}_{t}$ fixes the bundle over the section at infinity, and hence must act trivially on sections of the bundle over nonpolar fibres of $\widetilde{T P}^{1}$ (over which the bundle is trivial). Note that $Y$ is a time-translation-independent set, because isomorphic bundles over $\mathbb{P}^{1}$ have the same holomorphic structure.

It's not hard to see that if $\mathcal{V} \rightarrow \widetilde{T P}^{1}$ was constructed from a uniton as in $\S \S 3-7$, then we have just reconstructed the original framing of the trivial bundle $\underline{\mathbb{C}}^{N}$. 


\subsection{Time Invariance of $\nabla$ and $\Phi$.}

The connection $\nabla$ was defined by constructing covariant constant frames along null lines in $\mathbb{C}^{3}$. Consider again the covariant constant frame given by evaluation of sections over $G_{y}$ at $\left(\lambda_{0}, \eta_{0}\right)$, which defines a connection on a null plane through $y$. Translating by $t$, we get a null plane through $y+t$, the set of sections of $T \mathbb{P}^{1}$ through $\left(\lambda_{0}, \eta_{0}+2 \lambda_{0} t\right)$. By definition, the covariant constant frame is carried by $\tilde{\delta}_{t}$ into another covariant constant frame.

Specifically, a covariant constant frame over $\Pi_{\lambda_{0}}$ is given as the inverse image of a frame $f$,

$$
f \in \mathcal{V}\left(\lambda_{0}, \eta_{0}\right) \stackrel{\text { eval }}{\longleftarrow} H^{0}\left(G_{y}, \mathcal{V}\right)=\mathcal{W}_{y},
$$

for all $y$ such that $\left(\lambda_{0}, \eta_{0}\right) \in G_{y}$. Since

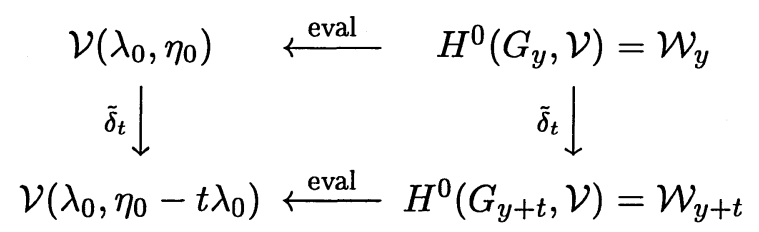

commutes, covariant constant frames are sent to covariant constant frames. Hence the null connections are invariant under $\tilde{\delta}_{t}$. Now $\nabla$ and $\Phi$ are defined in terms of these connections, so they must perforce be invariant. In terms of the special trivialisation, $\Psi$, the connection matrices and the matrix representing $\Phi$ are independent of $t$.

\subsection{Reality.}

It is sufficient to show that the constructed connection and Higgs' field satisfy our reality condition on a dense subset of $Y$. For simplicity we choose to work on $Y \cap \mathbb{C}^{3}$. We can also assume that $\mathcal{V}$ comes from $(\mathcal{W}, \nabla, \Phi)$ which are independent of time. It remains to show that they are real given a real structure on $\mathcal{V}$.

We assume that the principal bundle of frames of $\mathcal{V}$ comes with a fixed antiholomorphic involution, $\tilde{\sigma}$, lifting $\sigma$ which is given in a unitary frame as $X \mapsto \bar{X}^{t-1}$ on the fibres of fix $(\sigma)$. As was true for $\tilde{\delta}_{t}$ and $\mathcal{W}, \tilde{\sigma}$ induces a map on the bundle of frames of $\mathcal{W}, \operatorname{Fr}(\mathcal{W}) \cong \mathrm{GL}(N)$ :

$$
\operatorname{Fr}\left(\mathcal{W}_{y}\right)=H^{0}\left(G_{y}, \operatorname{Fr}(\mathcal{V})\right) \stackrel{\tilde{\sigma}}{\leftrightarrow} H^{0}\left(G_{\sigma(y)}, \operatorname{Fr}(\mathcal{V})\right)=\operatorname{Fr}\left(\mathcal{W}_{\sigma(y)}\right),
$$

which acts on a section, $f(y) \in H^{0}\left(G_{y}, \operatorname{Fr}(\mathcal{V})\right)$, by $f \mapsto \tilde{\sigma} \circ f \circ \sigma$, giving another section $f^{\prime} \in H^{0}\left(G_{\sigma(y)}, \operatorname{Fr}(\mathcal{V})\right)$. If $f$ is holomorphic, so is its 
image, which is the composition of one holomorphic and two antiholomorphic maps. The same argument as for $\tilde{\delta}_{t}$ similarly shows that $\tilde{\sigma}$ pulls back constant frames of the null connection on $\Pi$ to constant frames of the null connection on $\sigma \Pi$, and hence the null connections back to corresponding null connections.

In particular, if $\sigma(y)=y$, then a frame $f$ gets sent to $\tilde{\sigma}(f)$ where $\left.\tilde{\sigma}(f)\right|_{y}=$ $\overline{\left(f_{y}\right)}{ }^{t-1}$ in any unitary basis. So if $f$ is a covariant constant frame for $\nabla=$ $d+A$ in direction $X$, then $\tilde{\sigma}(f)$ is covariant constant for $\nabla$ in direction $\sigma(X)$ so

$$
\begin{aligned}
\left.A_{X}\right|_{y} & =\left.\frac{\partial}{\partial X} f \cdot f^{-1}\right|_{y} \\
\left.A_{\sigma(X)}\right|_{y} & =\left.\frac{\partial}{\partial \sigma(X)} \tilde{\sigma}(f) \cdot \tilde{\sigma}(f)^{-1}\right|_{y} \\
& =-\left.\frac{\partial}{\partial \overline{\sigma(X)}} f \cdot f^{-1}\right|_{y} \\
& =-\left.\overline{A_{\overline{\sigma(X)}}}\right|_{y} .
\end{aligned}
$$

Since $\sigma\left(\frac{\partial}{\partial x}\right)=\frac{\partial}{\partial x}, \sigma\left(\frac{\partial}{\partial y}\right)=\frac{\partial}{\partial y}$ and $\sigma\left(\frac{\partial}{\partial t}\right)=-\frac{\partial}{\partial t}$ (see (1.9)) we have

$$
A_{x}=-\bar{A}_{x}^{t}, \quad A_{y}=-\bar{A}_{y}^{t}, \quad A_{t}=\bar{A}_{t}^{t} .
$$

Reality for $\Phi$ follows in a similar fashion. To sum up,

$$
A_{x}, A_{y}, i A_{t}, i \Phi \in \mathrm{u}(N)
$$

in the chosen trivialisation of $\mathcal{W}$.

The proof of Theorem A will be complete once we have derived the energy formula in the next section.

\section{Chern Class equals Energy.}

We now use the interpretation of the extended solution to show that the second Chern class of the uniton bundle, evaluated on the fundamental cycle of $\widetilde{T P}^{1}$ is (a constant multiple of) the energy of the corresponding uniton.

The Chern-Weil homomorphism tells us that the the second Chern class of a bundle is given by integrating the trace of the curvature squared (wedged with itself) over the base manifold for any connection. Define a global 
connection on $\widetilde{T P^{1}}$ by gluing together two flat connections associated to particular gauges over

$$
\begin{aligned}
& U_{1}=\{0 \neq \lambda \neq \infty\} \subset \widetilde{T \mathbb{P}^{1}}, \text { and } \\
& U_{2}=\{|\lambda| \neq 1\}
\end{aligned}
$$

Over $U_{1}$ we take the $G_{\infty}$ trivialisation. Over $U_{2}$ we can use the constant trivialisation of $\mathbb{C}^{N} \times \mathbb{R}^{3} \rightarrow \mathbb{R}^{3}$ as follows:

The composition

$$
\mathbb{R}^{2} \times \mathbb{P}^{1} \cong\{t=0\} \hookrightarrow \mathbb{R}^{3} \times \mathbb{P}^{1} \rightarrow T \mathbb{P}^{1}
$$

is a (local) isomorphism onto $U_{2}$. Restriction to $\{t=0\}$ of the pullback of $\mathcal{V}$ by $\mathbb{R}^{3} \times \mathbb{P}^{1} \rightarrow T \mathbb{P}^{1}$ lifts the local isomorphism to a bundle isomorphism

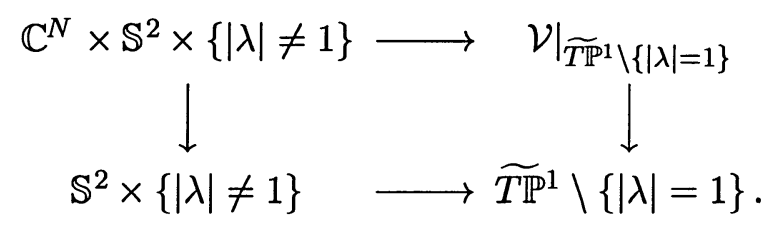

We define $\nabla_{2}$ to be the connection which annihilates the constant gauges of $\mathbb{C}^{N} \times \mathbb{S}^{2} \times\{|\lambda| \neq 1\} \rightarrow \mathbb{S}^{2} \times\{|\lambda| \neq 1\}$. (Note that this gauge cannot be holomorphic.)

The total connection is

$$
\nabla=(1-\rho) \nabla_{1}+\rho \nabla_{2}
$$

where $\rho$ is a smooth function $\rho: \mathbb{P}^{1} \rightarrow[0,1]$ depending only on $|\lambda|^{2}=\lambda \bar{\lambda}$ which is one on neighbourhoods of $\lambda \in\{0, \infty\}$ and zero on a neighbourhood of $\{|\lambda|=1\}$. Since $\nabla_{1}$ is flat, we can integrate over $U_{2}$ rather than all of $\widetilde{T P^{1}}$. Reality allows us to integrate over one of the components of $U_{2}$ and double our answer.

On $U_{2}, \nabla_{2}=d$ in the constant gauge over $\{t=0\}$. Since the $G_{\infty}$ trivialisation comes from the extended solution, $E_{\lambda}$, lifted to the total twistor space,

$$
\begin{gathered}
\nabla_{1}=d+E_{\lambda}^{-1} d E_{\lambda} \\
\stackrel{\text { def }}{=} d+B .
\end{gathered}
$$


The equations for the extended solution give

$$
\begin{aligned}
B_{\lambda} & =E_{\lambda}^{-1} \frac{\partial}{\partial \lambda} E_{\lambda} \\
B_{\bar{\lambda}} & =0 \\
B_{z} & =(1+1 / \lambda) A_{z} \\
B_{\bar{z}} & =(1+\lambda) A_{\bar{z}} .
\end{aligned}
$$

So

$$
\nabla=d+(1-\rho) B
$$

We calculate

$$
\Omega=\left(\rho^{2}-\rho\right) B \wedge B-d \rho \wedge B
$$

and

$$
\begin{aligned}
\operatorname{tr} \Omega \wedge \Omega= & \left(\rho^{2}-\rho\right)^{2} \operatorname{tr} B \wedge B \wedge B \wedge B \\
& -\left(\rho^{2}-\rho\right) d \rho \wedge \operatorname{tr} B \wedge B \wedge B-d \rho \wedge d \rho \wedge \operatorname{tr} B \wedge B \\
= & 0+3 \lambda\left(\rho^{2}-\rho\right) \rho^{\prime} \operatorname{tr}\left[B_{z}, B_{\bar{z}}\right] B_{\lambda} d z d \bar{z} d \lambda d \bar{\lambda}+0 \\
= & 3(1+\lambda)^{2}\left(\rho^{2}-\rho\right) \rho^{\prime} \operatorname{tr} \frac{\partial}{\partial \bar{z}} A_{z} B_{\lambda} d z d \bar{z} d \lambda d \bar{\lambda}
\end{aligned}
$$

The first term is zero because $B$ has no $d \bar{\lambda}$ component; the last term because $d \rho \wedge d \rho$ is a multiple of $d \lambda \wedge d \bar{\lambda}$ and $\operatorname{tr}\left[B_{z}, B_{\bar{z}}\right]=0$.

Lemma 11.1. For $\lambda \in \mathbb{C}^{*}$ fixed,

$$
\int_{z \in \mathbb{C}} \operatorname{tr} \frac{\partial}{\partial \bar{z}} A_{z} B_{\lambda} d z d \bar{z}=\lambda^{-1} \int_{z \in \mathbb{C}} \operatorname{tr} A_{z} A_{\bar{z}} d z d \bar{z}
$$

Proof. Integrate by parts to obtain,

$$
\int_{|z|<R} \operatorname{tr} \frac{\partial}{\partial \bar{z}} A_{z} B_{\lambda} d z d \bar{z}=-\int_{|z|=R} \operatorname{tr} B_{\lambda}\left(A_{z} d z\right)-\int_{|z|<R} \operatorname{tr} A_{z} \frac{\partial}{\partial \bar{z}} B_{\lambda} d z d \bar{z}
$$


Since $A_{z} d z=A_{\hat{z}} d \hat{z}(\hat{z}=1 / z)$ and $B_{\lambda}$ is a continuous function of $\mathbb{S}^{2}$, the line integral vanishes as $R \mapsto \infty$, leaving

$$
\begin{aligned}
\int_{z \in \mathbb{C}} \operatorname{tr} \frac{\partial}{\partial \bar{z}} & A_{z} B_{\lambda} d z d \bar{z}=-\int_{z \in \mathbb{C}} \operatorname{tr} A_{z} \frac{\partial}{\partial \bar{z}} B_{\lambda} d z d \bar{z} \\
= & -\int_{z \in \mathbb{C}} \operatorname{tr} A_{z}\left(\frac{\partial}{\partial \lambda} B_{\bar{z}}+\left[B_{\lambda}, B_{\bar{z}}\right]\right) d z d \bar{z} \quad(d+B \text { is flat }) \\
= & -\int_{z \in \mathbb{C}} \operatorname{tr} A_{z} \frac{\partial}{\partial \lambda}\left((1+\lambda) A_{\bar{z}}\right) d z d \bar{z} \\
& +(1+\lambda) \int_{z \in \mathbb{C}} \operatorname{tr}\left[A_{z}, A_{\bar{z}}\right] B_{\lambda} d z d \bar{z} \\
= & -\int_{z \in \mathbb{C}} \operatorname{tr} A_{z} A_{\bar{z}} d z d \bar{z}+(1+\lambda) \int_{z \in \mathbb{C}} \operatorname{tr} \frac{\partial}{\partial \bar{z}} A_{z} B_{\lambda} d z d \bar{z}
\end{aligned}
$$

(by (1.7)) which implies the required result.

Finally, we have to integrate over $\lambda$,

$$
\begin{aligned}
\frac{1}{2} \int \operatorname{tr} \Omega \wedge \Omega=3 \int \operatorname{tr}(1+\lambda)^{2}\left(\rho^{2}-\rho\right) \rho^{\prime}\left(\lambda^{-1} A_{z} A_{\bar{z}}\right) d z d \bar{z} d \lambda d \bar{\lambda} \\
=3 \int_{z \in \mathbb{C}} \operatorname{tr} A_{z} A_{\bar{z}} d z d \bar{z} \\
\cdot 2 i \int\left(\rho^{2}\left(r^{2}\right)-\rho\left(r^{2}\right)\right) \rho^{\prime}\left(r^{2}\right)\left(\frac{e^{-i \theta}}{r}+2 r+r e^{i \theta}\right) d r d \theta \\
=2 \pi i \int_{z \in \mathbb{C}} \operatorname{tr} A_{z} A_{\bar{z}} d z d \bar{z}
\end{aligned}
$$

So

$(11.2) \quad c_{2}(\mathcal{V})=\frac{-1}{4 \pi^{2}} \int_{\widetilde{\mathbb{P}^{1}}} \operatorname{tr} \Omega \wedge \Omega=\frac{-i}{\pi} \int_{z \in \mathbb{C}} \operatorname{tr} A_{z} A_{\bar{z}} d z d \bar{z}=\frac{1}{4 \pi} \operatorname{energy}(S)$

which implies that the energy spectrum is contained in $8 \pi \mathbb{Z}$, as shown by Valli [19].

\section{Ward's Construction.}

We are now ready to describe the link with the construction of Ward. The value of this is that Ward's construction involves only the factoring of a transition matrix (i.e. solving the Riemann-Hilbert problem) and no 
differential equations. In addition to its metaphysical significance, this result allows us to affirm the conjecture of Wood that unitons have rational functions in $x$ and $y$ as entries.

Let $\mathcal{V} \rightarrow \widetilde{T \mathbb{P}^{1}}$ be a uniton bundle. Theorem A allows us to assume that $\mathcal{V}$ was constructed from a uniton, $S$, via $(\nabla, \Phi)$, a solution to the Bogomolny equations. We are trying to find some intrinsic definition for $S$ on $\mathbb{R}^{3} \times \mathbb{P}^{1}$ which can be pushed down to $\widetilde{T P^{1}}$ and interpreted as a construction for $S$. We will in fact construct the extended solution $E_{\lambda}$ of Uhlenbeck.

Recall the twistor fibration

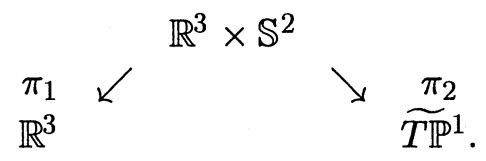

We defined the $G_{\infty}$ trivialisation of $\left.\mathcal{V}\right|_{\{0 \neq|\lambda| \neq \infty\}}$ by pulling back the extended solution $E_{\lambda}: \mathbb{S}^{2} \times \mathbb{C}^{*} \rightarrow G l(N)$ by

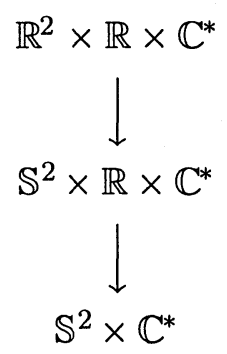

to give a solution to $\left\{\frac{\partial}{\partial t}, \bar{\nabla}, \nabla_{u}-i \Phi\right\}$. The $G_{\infty}$ trivialisation is the holomorphic section of $\left.\mathcal{V}\right|_{\{0 \neq \lambda \neq \infty\}}$ represented by this solution. On the other hand, a frame of $\mathcal{W}$ over a point, $y \in \mathbb{R}^{3}$, lifts to a trivialisation of $\pi_{1}^{*} \mathcal{W}$ restricted to the complex line $y \times \mathbb{S}^{2}$. Since this trivialisation is, by definition, in the kernel of $\bar{\nabla}$, it pushes down to a trivialisation of $\left.\mathcal{V}\right|_{G_{y}}$.

Now we use basic holomorphic geometry to reconstruct the extended solution. Since holomorphic functions on $\mathbb{P}^{1}$ are constant, holomorphic frames of $\mathbb{C}^{N} \times \mathbb{P}^{1} \rightarrow \mathbb{P}^{1}$ are determined by evaluation at a point. Since $\left.\mathcal{V}\right|_{G_{\infty}}$ and $\left.\mathcal{V}\right|_{P_{\lambda}}, \lambda \neq 0, \infty$, are trivial, the $G_{\infty}$ trivialisation is intrinsically defined (by fixing the framing $\phi$ ), as is the trivialisation of $\left.\mathcal{V}\right|_{G_{y}}$ which agrees with the framing $\phi$ at $G_{y} \cap P_{-1}$. The change of gauge $\mathcal{E}\left(\lambda, G_{y}\right)$ which relates these two trivialisations at $(\lambda, \eta)$, expressing the $G_{\infty}$ trivialisation in terms of the $G_{y}$ trivialisation of $\left.\mathcal{V}\right|_{G_{y} \cap P_{\lambda}}$ is thus well defined. (Note that at $\lambda=-1$, $\mathcal{E}\left(-1, G_{y}\right)=\mathbb{I}$.) Lifting to the total twistor space, $\mathcal{E}\left(\lambda, G_{y}\right)$, is the gauge transformation from the $\pi_{2}$-pulled-back $G_{\infty}$ trivialisation to the $\pi_{1}$-pulledback standard gauge, and so $\mathcal{E}\left(\lambda, G_{y}\right)$ is the extended solution, $E_{\lambda}$. 


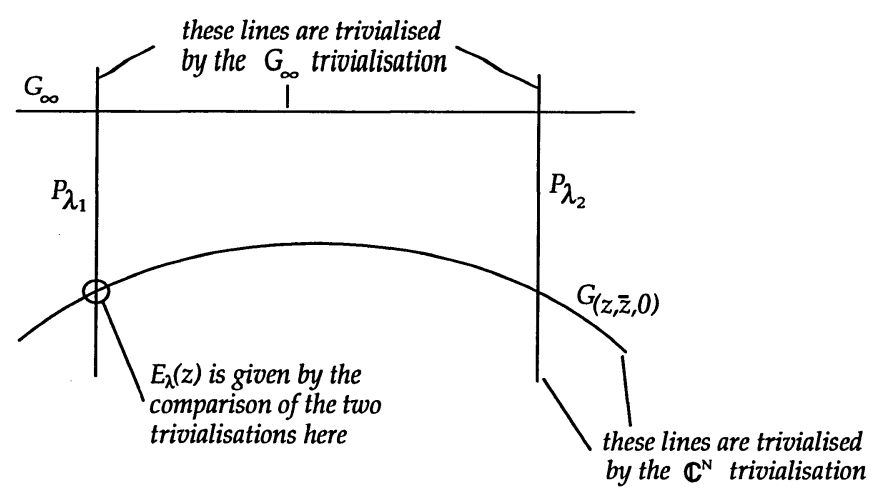

Figure 4: Compare the trivialisations at their point of intersection.

Although we have used the total twistor fibration to interpret the extended solution, we have only required the framing and the trivial holomorphic structure of $\mathcal{V}$ restricted to certain lines. We can thus express $E_{\lambda}$ intrinsically (without lifting to the total space) as the 'monodromy' around the cycle of $\mathbb{P}^{1}$ 's in Fig. 4.

To make this precise, what we are calling a 'monodromy' is actually the failure to commute of a cycle of homomorphisms given by the restriction map:

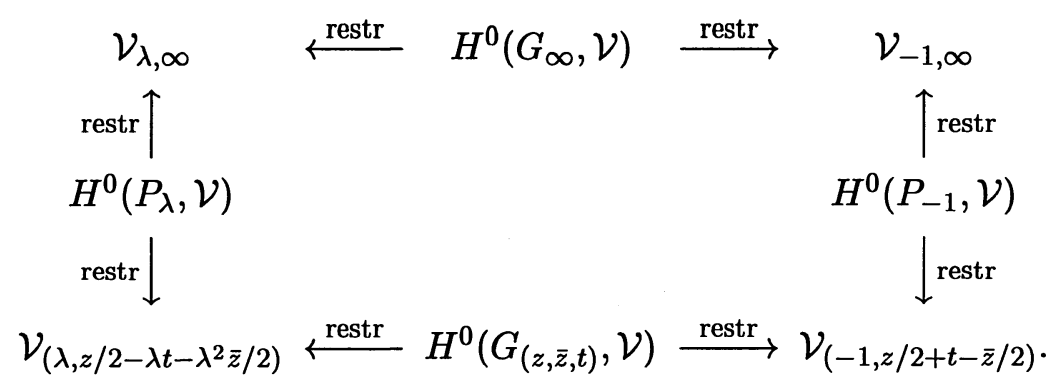

The 'monodromy' is independent of the choice of initial value, up to conjugation, as one would expect, since a change of framing of the bundle acts by conjugation on the uniton. We fix it by computing the 'monodromy' of the fixed frame $\phi \in H^{0}\left(P_{-1}, \operatorname{Fr}(\mathcal{V})\right)$. 


\subsection{Transition Functions.}

Ward's construction assumes the bundle is given by a transition matrix, so consider the covering of $\widetilde{T \mathbb{P}^{1}}$ given by

$$
\begin{aligned}
U & =\{\lambda \in \mathbb{C}, \eta \in \mathbb{C}\}, \\
\hat{U} & =\{\hat{\lambda} \in \mathbb{C}, \hat{\eta} \in \mathbb{C}\}, \\
U^{\prime} & =\left\{\lambda \in \mathbb{C}, \eta^{\prime} \in \mathbb{C}\right\}, \\
\hat{U}^{\prime} & =\left\{\hat{\lambda} \in \mathbb{C}, \hat{\eta}^{\prime} \in \mathbb{C}\right\} .
\end{aligned}
$$

The bundle $\mathcal{V}$ is determined by transition matrices $T, \hat{T}, T^{\prime}$ which map fixed frames of $\mathcal{V}$ over $U$ to $\hat{U}$, over $\hat{U}$ to $\hat{U}^{\prime}$, and over $U$ to $U^{\prime}$ respectively. Because $\mathcal{V}$ has certain triviality properties, we can choose the fixed frames such that

$$
\begin{aligned}
\left.\hat{T} T T^{\prime-1}\right|_{G_{\infty}} & =\mathbb{I}, \\
\left.T^{\prime}\right|_{P_{1}} & =\mathbb{I}, \text { and } \\
\left.\hat{T}\right|_{P_{-1}} & =\mathbb{I} .
\end{aligned}
$$

If the bundle is trivial when restricted to a complex line $\left(\mathbb{P}^{1}\right)$, then a framing above a point of the line extends uniquely to a nonvanishing frame on the line, because in this case evaluation

$$
H^{0}\left(\mathbb{P}^{1}, \underline{\mathbb{C}}^{N}\right) \stackrel{\text { eval }}{\longrightarrow} \mathbb{C}_{\text {point }}^{N}=\mathbb{C}^{N}
$$

is an isomorphism. We will think of this as defining a parallel translation within the line.

In terms of these frames parallel translation from a point on $P_{1}$ (in terms of the $U$ frame) to a point on $P_{-1}$ (in terms of the $\hat{U}$ frame) along $P_{1} \cup G_{\infty} \cup P_{-1}$ is given by $\mathbb{I}$. Since the bundle is trivial above real sections, we can split $T$, i.e. find analytic functions $H:\left\{(z, \bar{z}, t) \in \mathbb{R}^{3}, \lambda \in \mathbb{C}\right\} \rightarrow \mathrm{GL}(N)$ and $\hat{H}:\left\{(z, \bar{z}, t) \in \mathbb{R}^{3}, \lambda \in\left(\mathbb{P}^{1} \backslash\{0\}\right)\right\} \rightarrow \mathrm{GL}(N)$, such that

$$
T H_{\lambda}(z, \bar{z}, t)=\hat{H}_{\lambda}(z, \bar{z}, t) .
$$

Parallel translation from $P_{1} \cap G_{y}$ (in terms of the $\hat{U}$ frame) to $P_{-1} \cap G_{y}(U$ frame) along $G_{y}$ is given by

$$
H_{1}(y) \hat{H}_{-1}(y)^{-1}\left(=E_{1}(y)=S(y)\right),
$$


which gives the same formula for the uniton as in [21, 18] (up to a change of sign caused by a difference in basing conventions). One must verify that this doesn't depend on the choice of splitting.

Finally, we note that Ward actually takes two framings, along $P_{1}$ and $P_{-1}$. One framing would be equivalent to the restrictions (12.4). By taking two framings he does away with the basing condition. This is an important point if one wants to choose a different type of basing condition (other than $S(\infty)=\mathbb{I})$, to encode Grassmannian solutions for example.

\section{Example of a $U(3)$ uniton.}

Over a ruled surface, holomorphic bundles framed along a section of the ruling which are trivial on the generic line of the ruling are determined by their 'jumps' (i.e. the structure of the bundle on a neighbourhood of the 'jumping' lines on which the bundle is not trivial). (See [10], [11].) If $\eta$ is a coordinate on the lines and $\lambda$ parametrises the lines of the ruling, with $\lambda=0$ a jumping line, then the 'jump' is determined by a transition matrix $J\left(\lambda, \eta, \eta^{-1}\right)$ from $\{\eta \neq \infty\}$ to $\{\eta \neq 0\}$. It can then be 'glued' to the bundle over $\lambda \neq 0$ (which is trivialised by the framing of the bundle over a section of the ruling) by an additional matrix $M(\lambda)$. In our case, the $G_{\infty}$ trivialisation is the 'generic' trivialisation away from $\lambda=0$.

Assuming a 'unitary' choice of frame, $\phi$, the $G_{\infty}$ trivialisation is fixed by the real structure $(\tilde{\sigma})$ and the real structure exchanges the jumps at $P_{0}$ and $P_{\infty}$. So the uniton bundle is determined by the jump at $P_{0}, J\left(\lambda, \eta, \eta^{-1}\right)$, and its 'gluing', $M(\lambda)$, alone. As an example we will show how to construct a $U(3)$ uniton from such a jump.

Let

$$
\left(\begin{array}{ccc}
\eta^{2} & 2 \lambda \eta & \lambda^{2} \\
0 & 1 & \frac{\lambda}{\eta} \\
0 & 0 & \eta^{-2}
\end{array}\right)
$$

be the transition matrix for the jumping line $P_{0}$, and let this jump be glued in by $M(\lambda)=\mathbb{I}$ over the section at infinity to the trivial bundle on the union of the nonpolar fibres. For the bundle to admit a lift of the real structure, the transition matrix at $P_{\infty}$ must be conjugate to (13.1), i.e.

$$
\left(\begin{array}{ccc}
\hat{\eta}^{-2} & 0 & 0 \\
2 \hat{\lambda} & 1 & 0 \\
\hat{\lambda}^{2} & \hat{\lambda} \hat{\eta} & \hat{\eta}^{2}
\end{array}\right) .
$$


We have thus defined a bundle over $\widetilde{T P^{1}}$, with the required reality and triviality over fibres and the section at infinity. We need to check that it also has time translation and that it is trivial when restricted to real sections of $T \mathbb{P}^{1}$.

\section{2. $G_{\infty}$ trivialisation.}

Near $\lambda=0$, the $G_{\infty}$ trivialisation can be written as

$$
\left(\begin{array}{ccc}
0 & 0 & \lambda^{2} \\
0 & -1 & -\lambda \eta \\
\lambda^{-2} & 2 \frac{\eta}{\lambda} & \eta^{2}
\end{array}\right) \quad \text { and } \quad\left(\begin{array}{ccc}
1 & 0 & 0 \\
\frac{1}{\lambda \eta} & 1 & 0 \\
\frac{1}{\lambda^{2} \eta^{2}} & \frac{2}{\lambda \eta} & 1
\end{array}\right)
$$

in the $\{\eta \neq \infty\}$ and $\{\eta \neq 0\}$ trivialisations, respectively. (These are the sections' trivialisations of [10].)

Similarly, near $\hat{\lambda}=0$ the $G_{\infty}$ trivialisation can be written as

$$
\left(\begin{array}{ccc}
\hat{\eta}^{2} & \frac{\hat{\eta}}{\hat{\lambda}} & \hat{\lambda}^{-2} \\
-2 \hat{\lambda} \hat{\eta} & -1 & 0 \\
\hat{\lambda}^{2} & 0 & 0
\end{array}\right) \quad \text { and } \quad\left(\begin{array}{ccc}
1 & \frac{1}{\hat{\lambda} \hat{\eta}} & \frac{1}{\hat{\eta}^{2} \hat{\lambda}^{2}} \\
0 & 1 & \frac{2}{\hat{\lambda} \hat{\eta}} \\
0 & 0 & 1
\end{array}\right)
$$

We can use these trivialisations to check that our bundle admits time translation and is trivial on real sections.

\subsection{Time Translation.}

Time translation is determined by the requirement that it fixes the bundle above the infinity section. This means that it sends the framing

$$
\left(\begin{array}{ccc}
0 & 0 & \lambda^{2} \\
0 & -1 & -\lambda \eta \\
\lambda^{-2} & 2 \frac{\eta}{\lambda} & \eta^{2}
\end{array}\right) \quad \text { to } \quad\left(\begin{array}{ccc}
0 & 0 & \lambda^{2} \\
0 & -1 & -\lambda(\eta+\lambda t) \\
\lambda^{-2} & 2 \frac{(\eta+\lambda t)}{\lambda} & (\eta+\lambda t)^{2}
\end{array}\right)
$$

so it is

$$
\left(\begin{array}{ccc}
1 & 0 & 0 \\
-t & 1 & 0 \\
t^{2} & -2 t & 1
\end{array}\right)
$$

in terms of the first frame. Since this is continuous at $\lambda=0$, time translation extends as a continuous map to the jumping line.

Notice that on the big open set (and at $\eta=\infty$ ) the time translation acts trivially (in the $G_{\infty}$ trivialisation). 


\subsection{Real Triviality.}

Over the big open set $0 \neq \lambda \neq \infty$, different choices of global frames are related by $\mathrm{Gl}(3)$-valued functions of $(\lambda, \eta)$. We want functions which, when restricted to real sections, extend to $\lambda \in \mathbb{P}^{1}$. To test the extension we need to change to the appropriate frame over the open sets covering $P_{0}$ and $P_{\infty}$. The transition matrix restricted to $\eta=\infty$ is the gluing map (I), but close to $\eta=\infty$ it is given more generally by the sections' trivialisations over $\{\eta \neq 0\}$ :

$$
\left(\begin{array}{ccc}
1 & 0 & 0 \\
\frac{1}{\lambda \eta} & 1 & 0 \\
\frac{1}{\lambda^{2} \eta^{2}} & \frac{2}{\lambda \eta} & 1
\end{array}\right)_{P_{0}} \quad \text { and } \quad\left(\begin{array}{ccc}
1 & \frac{1}{\hat{\lambda} \hat{\eta}} & \frac{1}{\hat{\eta}^{2} \hat{\lambda}^{2}} \\
0 & 1 & \frac{2}{\hat{\lambda} \hat{\eta}} \\
0 & 0 & 1
\end{array}\right)_{P_{\infty}}
$$

respectively. Near $\eta=0$, we have to use the transition matrices to switch frames again, but this is the same as multiplying the original frame by the first sections' trivialisation there.

After some experimenting, we find that the frame

$$
\left(\begin{array}{ccc}
z^{2} & -\left(2 z+z^{2} \bar{z}\right) \lambda & \lambda^{2} \\
-\frac{z}{\lambda} & 1-z^{2} \bar{z}^{2} & 2 \bar{z} \lambda \\
\lambda^{-2} & \frac{2 z \bar{z}^{2}+\bar{z}}{\lambda} & \bar{z}^{2}
\end{array}\right)
$$

extends.

For example

$$
\begin{aligned}
& \left(\begin{array}{ccc}
0 & 0 & \lambda^{2} \\
0 & -1 & -\lambda \eta \\
\lambda^{-2} & \frac{2 \eta}{\lambda} & \eta^{2}
\end{array}\right)\left(\begin{array}{ccc}
z^{2} & -\left(2 z+z^{2} \bar{z}\right) \lambda & \lambda^{2} \\
-\frac{z}{\lambda} & 1-z^{2} \bar{z}^{2} & 2 \bar{z} \lambda \\
\lambda^{-2} & \frac{2 z \bar{z}^{2}+\bar{z}}{\lambda} & \bar{z}^{2}
\end{array}\right) \\
& =\left(\begin{array}{ccc}
1 & \lambda \bar{z}(2 \bar{z} z+1) & \lambda^{2} \bar{z}^{2} \\
-\frac{-z+\eta}{\lambda} & z^{2} \bar{z}^{2}-1-2 \eta \bar{z}^{2}-\eta \bar{z} & -2 \bar{z} \lambda-\lambda \eta \bar{z}^{2} \\
\frac{z^{2}-2 \eta z+\eta^{2}}{\lambda^{2}} & \frac{-2 z-z^{2} \bar{z}-2 \eta z^{2} \bar{z}^{2}+2 \eta+2 \eta^{2} \bar{z}^{2} z+\eta^{2} \bar{z}}{\lambda} & 1+4 \eta \bar{z}+\eta^{2} \bar{z}^{2}
\end{array}\right),
\end{aligned}
$$

which is seen to be holomorphic in $\lambda$ on the real section $\eta=z-\lambda^{2} \bar{z}$.

\subsection{Formula.}

The nice thing is that the frames (13.5) (in terms of the generic gauge) give us the transition from the $G_{(z, \bar{z}, 0)}$ gauge into the $P_{\lambda} / G_{\infty}\left(\lambda \in \mathbb{C}^{*}\right)$ gauges, and we can thus calculate the uniton by Ward's method to be

$$
\begin{aligned}
E_{\lambda} & =(\text { framing at } \lambda)(\text { framing at }-1)^{-1} \\
& =\frac{1}{\left(1+z^{2} \bar{z}^{2}+\bar{z} z\right)\left(4 \bar{z} z+z^{2} \bar{z}^{2}+1\right)}
\end{aligned}
$$




$$
\begin{gathered}
\left\{\lambda^{-2}\left(\begin{array}{ccc}
0 & 0 & 0 \\
0 & 0 & 0 \\
4 \bar{z}^{3} z+z^{2} \bar{z}^{4}+\bar{z}^{2} & 4 \bar{z}^{2} z+z^{2} \bar{z}^{3}+\bar{z} & z^{2} \bar{z}^{2}+4 \bar{z} z+1
\end{array}\right)\right. \\
+\lambda^{-1}\left(\begin{array}{ccc}
0 & 0 & 0 \\
-z^{3} \bar{z}^{4}-4 z^{2} \bar{z}^{3} & -z^{3} \bar{z}^{3}-4 z^{2} \bar{z}^{2} & -z-4 \bar{z} z^{2} \\
-\bar{z}^{2} z & -\bar{z} z & -z^{3} \bar{z}^{2} \\
2 z^{2} \bar{z}^{4}+5 z \bar{z}^{3} & -2 z^{3} \bar{z}^{4}-z^{2} \bar{z}^{3} & -4 z^{3} \bar{z}^{3}-4 z^{2} \bar{z}^{2} \\
+2 \bar{z}^{2} & +2 z \bar{z}^{2}+\bar{z} & -z \bar{z}
\end{array}\right) \\
+\lambda^{0}\left(\begin{array}{ccc}
z^{4} \bar{z}^{4}+4 z^{3} \bar{z}^{3} & z^{4} \bar{z}^{3}+\bar{z} z^{2} & 4 \bar{z} z^{3}+z^{4} \bar{z}^{2} \\
+z^{2} \bar{z}^{2} & +4 z^{3} \bar{z}^{2} & +z^{2} \\
-z^{3} \bar{z}^{4}-2 z^{2} \bar{z}^{3} & z^{4} \bar{z}^{4}-2 z^{2} \bar{z}^{2} & +2 z^{4} \bar{z}^{3}+z^{3} \bar{z}^{2} \\
+z \bar{z}^{2}+2 \bar{z} & +1 & -2 z^{2} \bar{z}-z \\
\bar{z}^{2}+z^{2} \bar{z}^{4} & -2 \bar{z}^{2} z-2 \bar{z}^{4} z^{3} & z^{4} \bar{z}^{4}+\bar{z}^{3} z^{3} \\
+\bar{z}^{3} z & -2 z^{2} \bar{z}^{3} & +z^{2} \bar{z}^{2}
\end{array}\right) \\
+\lambda^{1}\left(\begin{array}{ccc}
-z^{3} \bar{z}^{3}-4 z \bar{z} & -2 z-z^{2} \bar{z}+2 z^{3} \bar{z}^{2} & 2 z^{4} \bar{z}^{2}+5 z^{3} \bar{z} \\
-4 z^{2} \bar{z}^{2} & +z^{4} \bar{z}^{3} & +2 z^{2} \\
+2 z^{2} \bar{z}^{3}+2 \bar{z} & -4 \bar{z}^{3} z^{3}-4 z^{2} \bar{z}^{2} & +2 \bar{z} z^{2}+2 \bar{z}^{3} z^{4} \\
+2 \bar{z}^{2} z & -4 \bar{z} z & +2 z^{3} \bar{z}^{2} \\
0 & 0 & 0
\end{array}\right) \\
+\lambda^{2}\left(\begin{array}{ccc}
\bar{z} z+1+z^{2} \bar{z}^{2} & -2 z^{3} \bar{z}^{2}-2 z-2 \bar{z} z^{2} & z^{2}+\bar{z} z^{3}+z^{4} \bar{z}^{2} \\
0 & 0 & 0 \\
0 & 0 & 0
\end{array}\right)
\end{gathered}
$$

As pointed out by Francis Burstall, it is easy to test for holomorphic (one uniton) solutions. Assume $S$ were a one uniton, corresponding to a projection $\pi$, then its extended solution would have the form

$$
E_{\lambda}=g(\lambda)\left(\pi-\lambda \pi^{\perp}\right)
$$

and

$$
\begin{aligned}
\left(\left.E_{\lambda}\right|_{z=0}\right)^{-1} E_{\lambda} & =\left.\left(\pi-\lambda \pi^{\perp}\right)^{-1}\right|_{z=0}\left(\pi-\lambda \pi^{\perp}\right) \\
& =\left(\left.\pi\right|_{z=0}-\left.\lambda^{-1} \pi^{\perp}\right|_{z=0}\right)\left(\pi-\lambda \pi^{\perp}\right)
\end{aligned}
$$

would contain no powers $\lambda^{-2}$ or $\lambda^{2}$. Since $E_{\lambda}$ as constructed above does give such terms, our example must be a two uniton.

\section{Wood's Conjecture.}

Noting that all known examples of unitons were matrices of functions rational in $x$ and $y$ (equivalently $z$ and $\bar{z}$ ), Wood conjectured that this is 
always the case $([23])$. While it is true that the Bogomolny solution $(\nabla, \Phi)$ constructed from a uniton bundle is algebraic we do not know that the integration

$$
S^{-1} d S=2\left(A_{z} d z+A_{\bar{z}} d \bar{z}\right)
$$

preserves rationality. Continuing $S$ analytically, or equivalently, integrating $A$, we can't even rule out multivaluedness if $A$ is holomorphic on nonsimplyconnected domains.

The concrete expression (12.5), however, shows that $S$ extends to $Y \cap \mathbb{C}^{3}$ (a Zariski open set), and using (12.5) and the jumping-line normal form for transition matrices (see [10] and [14] for proofs) we can prove Corollary B.

Remark 14.1. In [1] we give an obviously rational, explicit formula for $S$ in terms of monad data of a special type. If this could be extended to general monad data, and hence general unitons, this proof would not be necessary.

Proof of Corollary B. Since the solution is $t$-invariant, we can ignore the third dimension.

We want to show $E_{\lambda}(z, \bar{z})=\hat{H}_{\lambda}(z, \bar{z}) H_{-1}(z, \bar{z})^{-1}$ is a rational $\operatorname{gl}(N)$ valued function on $\left\{(z, \bar{z}) \in \mathbb{P}^{1} \times \mathbb{P}^{1}\right\}$. A function is rational iff it is meromorphic iff it is meromorphic when restricted to the sets of a covering of $\mathbb{P}^{1} \times \mathbb{P}^{1}$, and a function is meromorphic iff its only singularities are poles. Thus we can answer a global question with a local answer. This is an example of Serre's GAGA principle ([17]), which says that analytic objects on compact varieties are algebraic.

Consider the family of open sets

$$
\left\{U_{z_{0}}=\left\{(z, w) \in \mathbb{P}^{1} \times \mathbb{P}^{1}: z \neq z_{0}, w \neq \bar{z}_{0}\right\}_{z_{0} \in \mathbb{P}^{1}}\right\} .
$$

Any three sets cover $\mathbb{P}^{1} \times \mathbb{P}^{1}$. Since the uniton equations are conformally invariant, the space of conformal maps $\operatorname{conf}\left(\mathbb{S}^{2}, \mathbb{S}^{2}\right)$ acts on the space of unitons. Our family of open sets is the orbit of $\mathbb{C} \subset \mathbb{P}^{1} \cong \mathbb{S}^{2}$ under conformal changes $z \mapsto 1 /(z-a)$. Each such change of coordinates produces a different uniton and a different uniton bundle. Rather than showing that a single uniton is meromorphic on enough sets of the family, it is easier to show that all its transformations under $\operatorname{conf}\left(\mathbb{S}^{2}, \mathbb{S}^{2}\right)$ are meromorphic on $U_{\infty}$, the basic open set which corresponds to our choice of coordinates. It is sufficient to do this for an arbitrary uniton. Let $S$ be such a uniton.

The expression (12.5) defines $S$ on $\mathbb{R}^{2}$, but extends just as well to $\mathbb{C}^{2}$ with potential singularities at the jumping lines. To see that they are poles, 
pull back the transition matrix $T$ by the sections' map

$$
\begin{aligned}
& \varphi: \mathbb{C}^{2} \times \mathbb{P}^{1} \rightarrow T \mathbb{P}^{1} \\
& (z, \bar{z}) \times(\lambda) \rightarrow\left(\lambda, \eta=z-\bar{z} \lambda^{2}\right) .
\end{aligned}
$$

If $(z, \bar{z}, 0)$ represents a jumping line of type $\left(k_{1} \leq k_{2} \leq \cdots \leq k_{N}\right)$ (i.e. $\left.\left.\mathcal{V}\right|_{G_{(z, \bar{z}, t)}} \cong \mathcal{O}\left(k_{1}\right) \oplus \cdots \mathcal{O}\left(k_{N}\right)\right)$, then we can make a holomorphic change of frame on some neighbourhood of the point so that $T$ has the form

$$
T=\left(\begin{array}{ccccc}
\lambda^{-k_{1}} & & & & \\
& \ddots & & p_{j}^{i} & \\
& & \ddots & & \\
& 0 & & \ddots & \\
& & & & \lambda^{-k_{N}}
\end{array}\right), \quad p_{j}^{i}=\sum_{a=-k_{j}+1}^{-k_{i}-1} p_{j a}^{i}(z, \bar{z}) \lambda^{a}
$$

where $p_{j a}^{i}$ are holomorphic functions. A section of $\left.\mathcal{V}\right|_{y}$ is given by

$$
\left(u^{1}, \ldots, u^{N}\right)^{t}, \quad u^{j}=\sum_{a=0}^{\infty} u_{a}^{j} \lambda^{a}
$$

such that

$$
T(y, \lambda)\left(\begin{array}{c}
u^{1} \\
\vdots \\
u^{N}
\end{array}\right) \text { is holomorphic in } 1 / \lambda,
$$

which puts conditions on $\left\{u_{a}^{j}\right\}$. Expanding the columns of $T \cdot u$ in Laurent series in $\lambda$, the conditions come from the coefficients of positive terms in $\lambda$ $\left(\lambda^{i}, i>0\right)$, which we can see are linear in $u_{a}^{j}$.

The result is a matrix equation for $U=\left(u_{a}^{j}\right)$ of the form

$$
M\left(p_{j a}^{i}\right) U=0
$$

where the coefficients of $M$ are linear polynomials in the $p_{j a}^{i}$. Since the $p_{j a}^{i}$ are holomorphic in $z$ and $\bar{z}, M$ is a holomorphic function of $y$. The matrix $M$ can be reduced by eliminating coefficients $u_{a}^{j}$ which are completely determined by other coefficients. The reduced matrix $\Gamma(y)$ whose elements are now polynomials in the $p_{j a}^{i}$ has the property that

$$
h^{0}\left(G_{y}, \mathcal{V}\right)=\operatorname{corank} \Gamma(y)
$$


and $\Gamma(y)$ has maximal rank when $\left.\mathcal{V}\right|_{G_{y}}$ is trivial. We can parametrise the kernel of $\Gamma(y)$ in a punctured neighbourhood of a jump by rational polynomials in $p_{j a}^{i} .(\Gamma(y)$ is polynomial, but to parametrise the kernel we must invert a maximal rank submatrix of $\Gamma(y)$, introducing poles at the jump where the rank of $\Gamma$ drops.) From this parametrisation of the kernel we can reconstruct the holomorphic frames of $\left.\mathcal{V}\right|_{G_{y}}$ (as a function of $y$ ) by rational polynomials in the $p_{j a}^{i}$, i.e. with possible poles at the jumping line. Since the extended solution is constructed from these frames by algebraic operations, it is also meromorphic.

Acknowledgement. For advice on matters of mathematics and mathematical exposition, I am indebted to Professor J. C. Hurtubise.

\section{References.}

[1] C. K. Anand, Uniton Bundles, McGill Ph.D. thesis, 1994.

[2] M. F. Atiyah \& R. Bott, The Yang-Mills Equations over Riemann Surfaces, Phil. Trans. R. Soc. Lond. A, 308 (1982), 323-615.

[3] C. P. Boyer, J. C. Hurtubise, B. M. Mann and R. J. Milgram, The topology of instanton moduli spaces. I: The Atiyah-Jones conjecture, Annals of Math.(2), 137 (1993), 561-609.

[4] H. Grauert, Ein Theorem der analytischen Garbentheorie und die Modulräume komplexer Strukturen, Publ. Math. Inst. Hautes Études Sci. 5 (1960), 233-292.

[5] A. Grothendieck and J. Dieudonné, EGA III: Étude cohomologique des faisceaux cohérents, Publ. Math. Inst. Hautes Études Sci. 4 (1960).

[6] Phillip Griffiths and Joseph Harris, Principles of Algebraic Geometry, John Wiley \& Sons, New York, 1978.

[7] R. Hartshorne, Algebraic Geometry, Springer-Verlag, New York, 1987.

[8] N. J. Hitchin, Monopoles and Geodesics, Commun. Math. Phys. 83 (1982), 579-602.

[9] J. C. Hurtubise, Asymptotic Higgs' Field of a Monopole, Commun. Math. Phys. 97 (1985), 381-389.

[10] Instantons and Jumping Lines, Commun. Math. Phys. 105 (1986), 107-122. 
[11] J. C. Hurtubise and R. J. Milgram, The Atiyah-Jones Conjecture for Ruled Surfaces, to appear in Crelle.

[12] Serge Lang, Introduction to Differentiable Manifolds, Wiley Interscience, 1962.

[13] M. K. Murray, Non-Abelian Magnetic Monopoles, Commun. Math. Phys. 96 (1984), 539-565.

[14] S. J. H. New, Handwritten notes.

[15] K. Pohlmeyer, Integrable Hamiltonian systems and interactions through constraints, Comm. Math. Phys. 46 (1976), 207-221.

[16] J. Sacks and K. Uhlenbeck, The existence of minimal immersions of 2-spheres, Annals of Math. 113 (1981), 1-24.

[17] J.-P. Serre, Géométrie algébrique et géométrie analytique, Ann. Inst. Fourier, 6 (1955-56), 1-42.

[18] K. Uhlenbeck, Harmonic Maps into Lie Groups (Classical Solutions of the Chiral Model), J. Differential Geometry, 30 (1989) 1-50.

[19] G. Valli, On the energy spectrum of harmonic 2-spheres in unitary groups, Topology, 27 (1988), 129-136.

[20] , Interpolation theory, loop groups and instantons, J. Reine Angew. Math. 446 (1994), 137-163.

[21] R. S. Ward, Classical Solutions of the Chiral Model, Unitons, and Holomorphic Vector Bundles, Commun. Math. Phys. 123 (1990), 319-332.

[22] F. Warner, Foundations of differentiable manifolds and Lie groups, SpringerVerlag, 1983.

[23] J. C. Wood, Explicit construction and parametrisation of harmonic twospheres in the unitary Group, Proc. London Math. Soc. (3), 58 (1989).

ReCeived March 14Th, 1995.

Department of Mathematics and Statistics

MCGILL UNIVERSITY

Burnside Hall, 805 Sherbrooke O.

MontrÉAL QC H3A 2K6 CANADA

Mathematics Research Centre

UNIVERSiTy OF WARWICK, COVERTY CV4 7AL, UK

E-MAIL ADDRESS: ANAND@MATHS.WARWICK.AC.UK 